Cronfa - Swansea University Open Access Repository

This is an author produced version of a paper published in :

Nature Reviews Materials

Cronfa URL for this paper:

http://cronfa.swan.ac.uk/Record/cronfa32284

\title{
Paper:
}

García de Arquer, F., Armin, A., Meredith, P. \& Sargent, E. (2017). Solution-processed semiconductors for nextgeneration photodetectors. Nature Reviews Materials, 2, 16100

http://dx.doi.org/10.1038/natrevmats.2016.100

This article is brought to you by Swansea University. Any person downloading material is agreeing to abide by the terms of the repository licence. Authors are personally responsible for adhering to publisher restrictions or conditions. When uploading content they are required to comply with their publisher agreement and the SHERPA RoMEO database to judge whether or not it is copyright safe to add this version of the paper to this repository. http://www.swansea.ac.uk/iss/researchsupport/cronfa-support/ 


\section{Solution-processed semiconductors for next-generation photodetectors}

F. Pelayo García de Arquer ${ }^{1, \dagger}$, Ardalan Armin ${ }^{2, \dagger}$, Paul Meredith ${ }^{2, *}$, Edward H. Sargent ${ }^{1, *}$

${ }^{1}$ Department of Electrical and Computer Engineering, University of Toronto, 35 St George Street, Toronto, Ontario M5S 1A4, Canada

${ }^{2}$ Centre for Organic Photonics \& Electronics, School of Mathematics and Physics, The University of Queensland, St Lucia Campus, Brisbane 4072, Australia

Efficient light detection is central to modern science and technology. Today's photodetectors are based principally on photodiodes made from crystalline inorganic elemental semiconductors such as silicon, and also compound semiconductors such as III-Vs. Photodetectors comprised of solution-processed semiconductors have recently emerged as candidates for next-generation light sensing. They combine ease of processing, facile integration with CMOS, compatibility with flexible substrates, and encouraging progress in performance. Recent advances and remaining challenges in solution-processed photodetectors are reviewed herein from both a materials and device perspective. Hybrid phototransistors and their potential to decouple prior trade-offs in noise vs. gain vs. speed are discussed. We update on rapid advances in metal halide perovskite photodiodes and feature their recent application in narrowband filter-less photodetection. We conclude with a summary of emerging avenues enabled by synergistic combinations of materials and device engineering. 
Photodetection underpins medical X-ray imaging, ubiquitous visible light cameras, and emerging applications such as near-infrared (NIR) medical imaging and short-wave infrared (SWIR) surveillance and machine vision. Present-day photodetection relies primarily on semiconductors that - depending on their bandgap - are able to transduce photons of different energies into electrical signals for subsequent processing, image construction and storage. Historically, this has been enabled by the use of photodiodes or phototransistors made from crystalline inorganic semiconductors such as silicon or III-V compounds - materials employed throughout modern electronics and optoelectronics. Lithographically patterned arrays of these devices create image sensors (arrays of miniaturized photodetectors) through combination with standard back plane architectures such as complementary metal oxide semiconductors (CMOS).

The miniaturized integration of the inorganic semiconductor photoactive materials with readout circuitry (ROIC) complicates device design and poses significant limits on compactness and sensitivity. ${ }^{1}$ Commercially available image sensors rely on photodetectors combined with the ROIC through front side or back side integration, depending on the position of the active layer in the sensor stack. ${ }^{2}$ Furthermore, conventional inorganic semiconductors are broadband absorbers - they produce a broad spectral photoresponse. For colour imaging and colourselective sensing, this fact further demands input-side color filter arrays and/or wavelength cutoff filters, significantly complicating the architecture and fabrication, and also negatively impacting pure colour replication.

Low-temperature solution processed semiconductors are an emerging class of photoactive material that can be processed in an ink-form via wet chemistry. They are technologically attractive from a number of perspectives: ease of deposition from solution over potentially large areas using readily available manufacturing relevant techniques such as roll-to-roll, spray coating or doctor blading; an ability to be processed at low temperatures and under ambient 
conditions; and compatibility with multiple ancillary material types (contacts, transport layers, etc.) including flexible substrates (Fig. 1). This processing versatility also offers major advantages for next generation top-surface photodetection schemes. This approach allows for the direct incorporation of an ultrathin photoactive layer on top of the ROIC with complete fillfactors (the photoactive fraction of the pixel area) delivering more compact and sensitive imaging systems (Fig 1c-d). ${ }^{3}$ This is especially important in attempts to overcome the interconnection bottleneck inherent in the drive to further miniaturize sensor arrays whilst maintaining resolution and sensitivity.

In addition to the manufacturing benefits of solution processed semiconductors, advantages from the materials physics and chemistry points of view stem from the ability to tailor these materials' optoelectronic properties. Especially appealing for photodetection is the capability to modify and control the semiconducting optical gap and the electronic energetics. Solutionprocessed semiconductors include organics, metal halide perovskites and inorganic nanocrystals/quantum dots. Each possesses (to lesser or greater extents) optoelectronic tunability, and is capable of addressing a certain spectral range (Fig. 1d).

In organic semiconductors, the optical gap can be tuned by varying molecular structure and/or by controlling their solid-state assembly (morphology). In combination, these strategies allow spectral photodetection coverage from the ultra-violet (UV) to the NIR. Metal halide perovskites (MHPs) on the other hand, are hybrid organic-inorganic compounds with the basic perovskite unit cell structure as their name suggests. Compositional tuning of the organic and inorganic fractions allows for the modification of the semiconductor band edge from the visible through to the NIR. In contrast, quantum confinement in colloidal quantum dots (CQDs) allows tuning of their spectra response well into the infrared by merely adjusting the size of the nanocrystals during synthesis. Moreover, the band structure and electronic energy levels of the resulting photoactive materials can be tailored by surface chemistry engineering. 
Advances in controlling and understanding many of these materials science aspects have led to state-of-the-art performance in a selected number of applications, and even commercial deployment. $^{3}$

Organic semiconductors are arguably the most optoelectronically adaptable of these classes of materials by virtue of their almost limitless molecular diversity. In particular, they have been shown significant potential for achieving color discrimination through molecular ${ }^{4}$ and device architecture ${ }^{5}$ tuning, or utilization of both strategies in combination. ${ }^{6}$ Low noise, broadband visible-near IR light detection have also been reported.,

Inorganic CQDs with broader responses up to the short-wave IR have also been demonstrated..$^{9-11}$ These ultrasensitive photodetectors exhibit either strong intrinsic gain or fast photoresponse, but the simultaneous achievement of both characteristics remains largely unexplored.

Metal halide perovskites are a more recent addition to the solution processable semiconductor family. They possess advantageous properties derived from their organic and inorganic components such as large absorption coefficients, low exciton binding energy and high charge carrier mobility. ${ }^{12,13}$ Their rare collection of desirably properties makes them potentially suitable for photodetector applications.

Despite significant recent progress in utilizing solution processed semiconductors for photodetection, several grand challenges and opportunities remain to be addressed both from basic science and applied device engineering perspectives. Materials properties such as tunability can be leveraged in new device architectures to implement new functions such as multispectral sensing. The solution processability could be exploited to devise new materials, combinations and structures to overcome classic photodetection limitations associated with (for example) dark noise. It is also interesting to consider how conventional and new materials 
could be combined synergistically to create hybrid photodetectors with unrivalled performance.

This review covers recent advances, ongoing progress, and present-day challenges in solution processed photodetectors from both the materials and device perspectives. We begin by offering a broad view of the last decade of advances. We particularly address the potential of metal halide perovskite photodiodes - a current hot topic in optoelectronics. We discuss how hybrid phototransistor architectures allow unlocking of the conventional photodetection limits by decoupling noise, gain and speed. Finally, we conclude by covering the latest progress in narrowband filter-less multispectral photodetection, and by offering a view on the new exciting avenues enabled by synergistic combination of materials and device platforms.

\section{Photodetector architectures}

Semiconductor-based photodetectors can be categorized into photoconductors, photodiodes and phototransistors. ${ }^{14}$ In photoconductors, one type of charge carrier is recirculated through symmetrical contacts before recombination with the opposite charge occurs. An external voltage is applied to read out the photon-derived changes in conductivity, and gain can be achieved based on the multiple carrier recirculation. Photodiodes, on the other hand, rely on the presence of a built-in potential in some form of photo-junction that assists in extracting the photogenerated carriers. Often, an additional reverse bias is applied to increase the charge collection efficiency. Phototransistors are optoelectronic amplifying switches in which a gateprogrammable semiconducting channel can be optoelectronically modulated facilitating or hindering charge transport whilst simultaneously providing gain. 
Photodetector performance is conventionally measured in terms of several different metrics associated with efficiency, noise and speed (Table 1). A basic, standalone figure of merit that quantifies the strength of the photoresponse is the responsivity $(R)$, which relates the generated photocurrent (or photovoltage) to a given incident power. Gain $(G)$ exists when the number of extracted photocarriers per absorbed photon exceeds unity. The temporal response of a photodetector is typically characterized by its $3 \mathrm{~dB}$ bandwidth (the modulation frequency at which the photoresponse is half that under continuous illumination). This quantity depends on the carrier transit time $\left(t_{\mathrm{tr}}\right)$ and $R C$-time of the circuit.

$$
f_{-3 d B}^{-2}=\left(\frac{3.5}{2 \pi t_{\mathrm{tr}}}\right)^{-2}+\left(\frac{1}{2 \pi R C}\right)^{-2}
$$

It has been shown that in dispersive materials such as organic semiconductors, the relevant transit time defining the frequency responses the slower carrier's transit time. ${ }^{8}$

Another important quantity is the linear dynamic range (LDR), that measures the range $\left(L_{\mathrm{min}}\right.$ to $\left.L_{\max }\right)$ over which the photoresponse is linear with respect to the incident light irradiance. This can be expressed in terms of number of decades or logarithmically (dB). Both $\mathrm{LDR}=20 \cdot \log \left(L_{\max } / L_{\min }\right)$ and $\mathrm{LDR}=10 \cdot \log \left(L_{\max } / L_{\min }\right)$ are used to quantify the LDR in $\mathrm{dB}$. The latter is employed when the linearity of the power $v s$. voltage is under question. A careful comparison between the LDRs of different devices needs to be carried out to avoid the confusion between different logarithmic factors. ${ }^{7}$ Special attention needs to be taken when estimating the LDR from log-log plots since this could otherwise potentially result in its overestimation. ${ }^{15-20}$

Ultimately, the sensitivity of a photodetector is given by its ability to deliver enough signal to noise ratio (SNR) with respect to the noise spectral density $\left(S_{N}\right)$. The noise equivalent power (NEP) represents the incident power that would be required to yield a near-unity SNR over one Hertz bandwidth. 
The overall figure of merit for photodetection, which normalizes the NEP to the device area $(A)$ and electrical bandwidth of the measurement $(B)$, is the specific detectivity $\left(D^{*}\right) . D^{*}$ corresponds to the SNR when the detection bandwidth is $1 \mathrm{~Hz}$, device area $1 \mathrm{~cm}^{2}$ at an incident power of $1 \mathrm{~W}$, and can be written as

$$
D^{*}=\frac{R \sqrt{A_{d} B}}{S_{\mathrm{N}}}
$$

There are many sources that contribute to the noise current: thermal, shot, flicker $(1 / f)$ and generation-recombination components. ${ }^{21,22}$ We note that the noise current cannot be directly inferred from the shot noise (simply defined by the dark current) and thermal noise in disordered semiconducting systems. It has been found in organic semiconductors, MHP and CQD photodetectors, that the actual measured noise is larger than the RMS of shot and thermal noises. As such, inferring the $D^{*}$ from the shot noise can result in overestimation.

Below we briefly summarize the performance metrics of relevant photodetectors based on different materials and architectures (Table 2).

\section{Organic Semiconductor Photodetectors}

Electrical conductivity was discovered in doped polypyrrole ${ }^{23}$ in the 1960 s. During the 1970 s and 80 s, this initial discovery was followed by the creation of myriads of small molecule and polymeric conductors and semiconductors, culminating in the demonstration of multiple optoelectronic device platforms including organic solar cells, ${ }^{24}$ field effect transistors ${ }^{25}$ and ultimately photodetectors. ${ }^{26}$

The potential for organic photodetectors (OPDs) to achieve filter-less narrowband colour sensing was recognised early in the first homojunctions and linear heterojunctions. ${ }^{27}$ However, these devices were limited by the excitonic nature of organic semiconductors that typically exhibit exciton binding energies of order $100 \mathrm{~s}$ of $\mathrm{meV}$, severely impacting charge generation and hence, transport and external quantum efficiency (EQE). This problem has been largely 
resolved by employing the bulk heterojunction $(\mathrm{BHJ})$, a blend of two organic semiconductors with different chemical potential energies, the donor and the acceptor, to facilitate charge separation.

Efficient BHJ systems can exhibit EQEs in excess of $80 \%$ even at short circuit, and hence the majority of OPDs are based on BHJ photoactive layers in a diode architecture. Unfortunately, utilizing two mixed components in the photoactive layer significantly complicates the spectral response since both the donor and acceptor contribute to photogeneration. ${ }^{28}$ Additional optoelectronic strategies have to be implemented in order to produce a narrowband response in OPDs, and we will return to this later in the review.

Considering more broadly the performance of OPDs, it is first important to appreciate that organic semiconductors are electrically disordered. This leads to relatively low carrier mobilities for both carrier types, which in turn hinders photogenerated carrier collection and limits performance metrics such as EQE, LDR and temporal response. Low carrier mobilities also constrain the junction thickness to typically $<200 \mathrm{~nm}$, meaning higher leakage currents and hence dark noise, and also introducing absorption compromises.

Organic semiconductors with improved packing and stronger intermolecular interactions, have been synthesized that exhibit high hole mobilities on the order of $10^{-2} \mathrm{~cm}^{2} / \mathrm{Vs}$ in diodes. However, charge collection is still limited by the electron mobility of the fullerenes $\left(\sim 10^{-3}\right.$ $\mathrm{cm}^{2} / \mathrm{Vs}$ ), which remains the predominant acceptor. It is also worth noting that materials with improved transport should deliver thicker junctions and hence assist with reducing leakage and dark noise.

The optical gap of organic semiconductors can be tuned through modulating the conjugation length. Using this approach, broadband OPDs have been reported for various spectral bands across the UV to NIR. Visible-blind OPDs with high responsivity in the UV have been reported 
with large LDRs > 10 orders of magnitude and large gain. ${ }^{29}$ IR-blind broadband visible OPDs have also been demonstrated using small molecules ${ }^{30}$ and polymeric ${ }^{31}$ semiconductors. In addition, it has been shown that optically thick PCDTBT:PC70BM BHJs can deliver IR-blind and a spectrally flat photoresponse across the visible with a $D^{*}$ approaching $10^{13}$ Jones on large and flexible substrates. ${ }^{8}$ This has been also demonstrated in fully printed PCDTBT:PC70BM photodiodes. $^{32}$

OPD progress is strongly coupled to improvements in organic solar cells, in particular to the use of new materials with narrower optical gaps, ${ }^{33,34}$ and to advances in the understanding of device electro-optics manipulation. ${ }^{35,36}$ Spectral responses up to $1000 \mathrm{~nm}$ have been reported, ${ }^{30,31,37}$ but decreasing the optical gap of organic semiconductors to below $1 \mathrm{eV}$ is challenging. Extension of the spectral response of broadband OPDs to $1450 \mathrm{~nm}$ using the narrow gap polymer, poly(5,7-bis(4-decanyl-2-thienyl)-thieno $(3,4-b)$ diathiazole-thiophene2,5) blended with PC60BM has been reported. ${ }^{7}$ The mechanism by which a photoresponse can be generated past the donor gap is still to be resolved.

A different approach has been employed to extend the spectral bandwidth of photodetectors based upon P3HT:PCBM blends - previously typically sensitive only to visible light. Rauch et al. introduced $\mathrm{PbS}$ nanoparticles and achieved spectral responses beyond $1100 \mathrm{~nm}$ - an indication of charge/energy transfer from the nanoparticle to the polymer matrix - in a photodiode array prototype already integrated into a ROIC panel. ${ }^{38}$ The incorporation of $\mathrm{ZnO}$ in a similar nanocomposite fashion was reported by different groups to result in photoconductive gain. ${ }^{29,39,40}$ Dong et al. found that the incorporation of $\mathrm{ZnO}$ nanoparticles within P3HT:PCBM:PbS ternary blends could deliver large EQEs $(\sim 1000 \%) .{ }^{40}$ Hence, broadband OPDs with respectable performance metrics covering the UV to near-IR have been realized.

\section{Colloidal Quantum Dot Photodetectors}


The facile bandgap tunability of CQDs from the visible further into the infrared has long been appreciated as a means to extend the range of OPDs. Preliminary efforts employed CQDs as sensitizing agents of polymeric charge transporting matrices. ${ }^{41}$ Photophysical studies revealed that visible-light excitons in $\mathrm{CdS}$ could be transferred to the host matrix giving rise to photoconductivity. This notion was further elaborated in 2005 when CdSe visible photodetectors with an inferred $D^{*}$ of $10^{8} \mathrm{Jones}^{42}$ and short-wave IR PbS CQD photodetectors were both reported. ${ }^{43}$

A new paradigm in solution processed photodetection was demonstrated in 2006 , where the sequential, ordered processing of PbS CQDs led to photoconductive CQD solid films. ${ }^{9}$ The controllable fusing and oxidation of the CQDs delivered high photo-multiplicative gain as a consequence of minority carrier trapping, yielding gains in excess of $1000, R \sim 2700 \mathrm{~A} / \mathrm{W}$ and impressive measured $D^{*}$ up to $2 \cdot 10^{13}$ Jones in the short-wave IR. This set a major landmark for CQD photodetector sensitivity surpassing that of epitaxially deposited InGaAs counterparts, with the added manufacturing benefits of solution processed materials. The high photoconductive gain also offered new avenues for integrated in-pixel amplification, greatly simplifying sensor design. Visible photoconductors that exploit CQD bandgap tunability were subsequently reported. ${ }^{44}$

Controllable introduction of defects does lead to high gain and detectivity, but at the expense of a sub $20 \mathrm{~Hz}$ temporal response. Identification of associated trap states and surface chemistry strategies that enabled their selective passivation, has thereby allowed improvements in response times to below $\sim 25 \mathrm{~ms}$, but with reduced gain. Although sufficient for most imaging applications, this gain-bandwidth product limitation represents a significant drawback for higher speed applications such as in-flight object recognition. ${ }^{45,46}$ Further improvements in CQD films to address their most acute limitation, i.e. mobility, has led to the realization of 
faster photoconductive responses $\left(0.4 \mathrm{kHz}\right.$ bandwidth) with high internal gain $\left(G>6.5 \cdot 10^{3}\right)$ and detectivity $\left(D^{*} \sim 10^{13}\right.$ Jones $){ }^{47}$

The generation of multiple carriers per incident photon is also possible in CQDs through multiple exciton generation (MEG). This quantum mechanical-related phenomenon relies on the generation of multiple excitons when the energy of the incoming photon is several times greater than the semiconductor optical gap. The potential of this process has been explored in several material systems and architectures. ${ }^{4-52}$

The successful implementation of CQD solids into full photodiode architectures remained elusive until 2007, partly due to a limited understanding and control over energy levels, surface chemistry and density of defect states. ${ }^{53}$ Record sensitivity-bandwidth combinations, with a $D^{*}$ exceeding $10^{12}$ Jones in the short-wave IR and a $3 \mathrm{~dB}$ bandwidth up to $5 \mathrm{MHz}$, were achieved in Schottky PbS CQDs photodiodes; this was accomplished by a combination of improved carrier mobility via a more complete ligand exchange and a carrier extraction strategy that ensured drift collection dominated over diffusion. ${ }^{10}$ Heterojunction architectures based on the $p$ - $n$ geometry with wide bandgap metal oxides ${ }^{54}$ and with polymers ${ }^{38}$ or fullerene derivatives ${ }^{55}$ have also been reported with high detectivities.

The CQDs bandgap tunability can also be exploited to engineer graded energy landscapes designed to funnel photogenerated carriers with the aid of a graded electric field. ${ }^{56}$ These quantum-funnel photodiodes benefit from improved drift-assisted carrier extraction, yielding $\mathrm{MHz}$ 3dB-bandwidths even at zero bias and high detectivity in the near-IR.

The carrier extraction/absorption compromise that exists in CQD films gave rise to attempts to integrate photonic and plasmonic systems seeking to reduce the electrical volume of the photoactive material for a given absorption. This approach could result both in reduced carrier transit times, therefore in a faster photoresponse, and in lower dark currents and noise, 
ultimately leading to high sensitivities; this always provided the volume reduction compensates the increasing surface recombination and contact noise arising from the higher surface-tovolume ratio. Based on this view, improvements in responsivity ${ }^{57,58}$ and detectivity ${ }^{59}$ have been achieved by incorporating metallic nanostructures in the photodetector architecture. It was noted that special care should be taken in integrating these metallic motifs within the CQDs, as they can remotely modify the electrical properties of the QD solid film. ${ }^{60}$ Indeed, their sole electrical interaction was shown to result in improved photodetector characteristics through remote doping and passivation. ${ }^{61}$

In summary, CQD progress could be generally categorized by photoconductors that exhibit high gain but are slow, or photodiodes that were fast but lacked photo-multiplication. In the next sections we will describe how advances in material science and device architecture have potentially unlocked this trade off.

\section{Metal Halide Perovskite Photodetectors}

The advent of metal halide perovskites (MHP) has led to a major step forward in photovoltaics, and opened promising avenues for photodetection. Perovskites, in general, have a crystal unit cell of the type $\mathrm{ABX}_{3}$, where $\mathrm{B}$ is a cation and $\mathrm{X}$ is an anion, forming an octahedron $\left[\mathrm{BX}_{6}\right]^{4-}$ (Fig. 2a). The octahedra are stabilized by a second cation A; a simple and archetypal example of this structure is $\mathrm{CaTiO}_{3}$. In organohalide lead perovskites, the cation A is a small organic molecule, e.g., methylammonium $\left(\mathrm{CH}_{3} \mathrm{NH}_{3}{ }^{+}\right)$or formamidinium $\left(\mathrm{H}_{2} \mathrm{NCHNH}_{2}{ }^{+}\right)$, B is a metal such as lead or tin, and the $\mathrm{X}$ is a halide combination. The use of MHPs in optoelectronics is compelling: they can be solution processed from simple earth abundant precursors, ${ }^{13}$ or evaporated using relatively low deposition and evaporant temperatures just like organic semiconductors; they have energy bandgaps which are tunable with the halide ratio; and they can form efficient thin single photojunctions using simple planar or mesoporous scaffold-based

architectures due to their low exciton binding energy, ${ }^{62}$ similar to that of inorganic 
semiconductors. As such, MHPs potentially combine the advantages of inorganic and organic semiconductors. The non-excitonic behaviour of optimised MHP films results in the simplest diode architecture in which charges can be photogenerated and collected almost with no loss (Fig. 2b-c).

More than two years after the demonstration of the first efficient MHP solar cells by the Miyasaka ${ }^{63}$ and Snaith groups, ${ }^{64} \mathrm{CH}_{3} \mathrm{NH}_{3} \mathrm{PbI}_{3}$ was employed in photoconductors in $2014 .{ }^{65}$ However, the first low noise MHP photodiodes were reported in 2015 almost at the same time by four research groups, ${ }^{66-69}$ and the reason behind this relatively long delay is important: metal halide perovskites were initially deployed in metal oxide mesoporous scaffolds, which are not suitable for photodetection due to their large capacitances. Planar junctions became more common in $2014,{ }^{70}$ however solution processing of these junctions was not an easy task. Low noise photodetection requires very high quality and dense active layers to prevent leakage current. A reliable and reproducible solution processing method to form planar MHP layers was introduced late in 2014 thus enabling planar junction diodes. ${ }^{71}$

In the four seminal reports on MHP photodiodes where both inverted and conventional architectures were employed, PCBM and/or C60 were used as electron extracting and hole blocking interlayers. In all of these reports, $D^{*} \sim 10^{12}$ Jones and EQEs of 60 to $80 \%$ in the visible were achieved. Lin et al. ${ }^{66}$ showed that the response speed in these photodiodes is RCtime limited i.e., the second term of eq. 1 dominates, obtaining a response time of $120 \mathrm{~ns}$ as shown by Fang et al. ${ }^{69}$ This is predominantly because of the large dielectric constant of metal halide perovskites (70 to 30 from static to $1 \mathrm{MHz}$ frequencies). ${ }^{62}$ It has also been shown that the use of thick fullerene buffer layers can reduce the capacitance with no detrimental effect on the device performance and increasing the $3 \mathrm{~dB}$ response from $100 \mathrm{kHz}$ to $500 \mathrm{kHz}$. For small pixels, it has been shown that the response speed becomes carrier transit time limited and response speed could exceed MHz in MHP photodiodes. ${ }^{66,67,69}$ 
As with organic semiconductor photodiodes, the electro-optical properties of MHPs can be tuned by changing the composition of the perovskite material (Fig. 2d). Recently, Dong et al. employed $\mathrm{CH}_{3} \mathrm{NH}_{3} \mathrm{PbI}_{3}$ active layers in which the ratio of $\mathrm{PbI}_{2} / \mathrm{CH}_{3} \mathrm{NH}_{3} \mathrm{I}$ was engineered in a trap-engineered diode architecture. ${ }^{72}$ This yielded photoconductive gains up to 400 with a response speed on the order of $\mu$ s and $85 \mathrm{~dB}$ of linearity (Fig. 2e). ${ }^{72}$ Also similar to organic semiconductors, MHPs can be combined with inorganic nanoparticles. Liu et al. have recently reported on the use of $\mathrm{PbS}$ QDs in order to expand the spectral bandwidth of MHPs to the NIR. ${ }^{16}$ Lin et al. reported a new strategy to modulate MHPs optical and electrical properties based on molecular doping. ${ }^{73}$

Recently, MHPs also attracted attention for X-ray detection. ${ }^{74,75}$ Due to the large atomic number of lead, its compounds exhibit large X-ray absorption coefficients. This led to the study of semiconducting lead halides as potential candidates for X-ray detectors. ${ }^{76}$ This feature, combined with the high mobilities and low defect density of organo lead halide perovskites, led to the realization of fast and high gain (over $10^{4}$ carriers per photon) X-ray photodetectors. One of the challenges of this approach is the relatively large junction thickness, on the order of $\sim \mathrm{mm}$, required to absorb a significant fraction of X-ray photons impinging on the device. This demands long high-diffusion lengths in order to avoid excessive recombination. Single crystal methylammonium lead tribromide perovskite detectors have been recently reported that, in view of record mobility-lifetime products $\left(\sim 1 \cdot 10^{-2} \mathrm{~cm}^{2} \mathrm{~V}^{-1}\right)$ and small surface charge recombination velocity, showcase sensitivities exceeding that of standard amorphous selenium detectors $\left(80 \mu \mathrm{C} \mathrm{Gy}^{-1} \mathrm{~cm}^{-2}\right){ }^{77}$

In summary, MHPs have emerged with significant potential and impact for optoelectronics and in particular photodetection. There are, however, many unanswered questions regarding their basic operational principles such as dielectric relaxation and ionic behavior, and the interrelation of these phenomena with photogeneration and collection. In the next section we 
examine how advances in device design with the introduction of hybrid phototransistor architectures can overcome traditional photodetection trade-offs regarding noise, photoresponse and speed.

\section{Phototransistors}

In phototransistors, the electrical properties of a charge transporting channel are modulated with light. There are a number of phototransistor architectures. Metal-oxide semiconductor (MOS) transistors are three terminal devices that consist of a metallic gate (typically $\mathrm{Si}$ ), an oxide insulator (typically $\mathrm{SiO}_{2}$ ), and a photoactive channel fabricated atop contacted with drain and source electrodes. The advent of solution processable materials enabled the introduction of thin film phototransistors (TFTs) that overcome the rigidity limitations of crystalline Sibased devices. Initial TFTs mainly relied on organic materials to define the photoactive channel, ${ }^{26}$ which allowed their integration with flexible substrates. ${ }^{78}$ Despite this particular advantageous property, the performance of these detectors has remained modest largely due to poor carrier mobilities in the photoactive channel.

Progress was reported in 2012 by Konstantatos et al., who used a hybrid combination of solution processed materials with high conductivity $2 \mathrm{D}$ materials. ${ }^{79}$ In this type of device, photosensitization and transport take place through separated optimized phases: one that enables efficient light absorption and one that provides with fast charge recirculation. The combination of $\mathrm{PbS}$ CQDs and graphene resulted in ultrahigh gain $\left(G \sim 10^{8}\right.$ electrons per photon) and exceptional responsivities for short-wave IR photodetection. The CQD film was assembled and crossed-linked to the graphene transport layer with 1,2-etanedithiol ligands, which facilitated charge injection into the graphene, and also provided electron trap states yielding a large photoconductive gain. The measured $D^{*} \sim 7 \cdot 10^{13}$ Jones represented a significant step forward for visible-short-wave IR imaging, yet is limited by the $\sim 10 \mathrm{~ms}$ temporal response. 
The enhanced performance of these hybrid devices is based on the separation of photosensitization (photoactive layer) and electrical transport (transport layer) (Fig. 3a). If appropriately gate biased $\left(\mathrm{V}_{\mathrm{g}}\right)$ the transport channel will be closed in the dark (Fig. 3b). Under illumination, photogenerated charges separate promoted by the internal electrical field existing at the gated junction, and one type of carrier (say for example holes) will be injected into the transport channel (Fig. 3c). This charge carrier can recirculate several times before a lowermobility opposite carrier does likewise (or else becomes trapped) (Fig. 3d). The photoconductive gain under such conditions is given by,

$$
G=\frac{t_{\text {rec }}}{t_{\text {transit }}}=\frac{t_{\text {rec }}}{L / v} \quad \text { (eq. 2) }
$$

where, $t_{\text {rec }}$ is the time required by the captured charge to be released and recombine in the sweeping electrodes, $L$ is the channel length and $v$ is the velocity of the injected charge. The excellent mobility of 2D semiconductors such as graphene $\left(\sim 10^{5} \mathrm{~cm}^{2} / \mathrm{Vs}\right.$ at room temperature $)$ therefore enables extraordinarily high photomultiplicative gains; and it does so without limiting the temporal response of these devices, which will be given by the slower of charge injection and recombination.

In a similar fashion, other authors have reported implementation of solution processed materials with graphene in hybrid phototransistors. Large area and flexible infrared detectors with excellent stability were reported by Sun et al. ${ }^{80}$ based upon PbS CQDs solid films cast and assembled with pyridine ligands on top of chemically vapour deposited (CVD) graphene achieving $R \sim 10^{7} \mathrm{~A} / \mathrm{W}$. Metal halide perovskite-graphene phototransistors have also been reported, where $\mathrm{CH}_{3} \mathrm{NH}_{3} \mathrm{PbBr}_{2} \mathrm{I}$ provide visible sensitization. ${ }^{81,82}$ We note that pure MHP phototransistors based on $\mathrm{CH}_{3} \mathrm{NH}_{3} \mathrm{PbI}_{3-\mathrm{x}} \mathrm{Cl}_{\mathrm{x}}$ perovskites have also been explored, that exhibited ambipolar behaviour with sub $10 \mu$ s time response operation but gains below $10^{3}{ }^{83}$ 
The absence of bandgap in graphene precludes however full exploitation of this hybrid architecture since the transport channel cannot be totally closed, which ultimately results in significant dark currents. Hence, the use of other high mobility gapped materials is a possibility, and the emergence of 2D materials with exceptional transport properties and visible bandgaps such as $\mathrm{MoS}_{2}$ has allowed for further development in the field. In $2014 \mathrm{PbS}$ CQDs-MoS 2 hybrid phototransistors were reported by Kufer et al, ${ }^{84}$ which benefited from significantly lower dark currents (over an order of magnitude) whilst maintaining high responsivities. The introduction of interface states as a consequence of the chemical modification of the transport layer was found to deteriorate noise and time response characteristics, and major improvements can be expected provided these could be controlled. ${ }^{85}$

The incorporation of solution processable materials with other high mobility materials in nanocomposites has also been explored. Wang and coworkers achieved hybrid phototransistors with high responsivities $\left(R \sim 10^{4} \mathrm{~A} / \mathrm{W}\right)$ and sub-ms response times based on methylammonium lead trihalide perovskites / single-walled carbon nanotubes hybrids. ${ }^{86}$ The resulting detectivity of these visible-NIR ambipolar phototransistors is reported to be above $10^{14}$ Jones. Other hybrid phototransistor architectures based on the combination of $\mathrm{ZnO} \mathrm{NCs}, \mathrm{PbS}$ CQDs and organic semiconductors were reported with UV-IR photon counting capabilities. ${ }^{87}$

The separation of transport and sensitization can also be monolothically achieved in much simpler architectures. Photojunction Field Effect Transistors (photoJFETs) rely on the presence of a high resistance depletion region between a pair or source and drain non-ohmic contacts (Fig. 3e). Under dark conditions, this ensures a minimum noise level (Fig. 3f). When illuminated, photogenerated charges split based on the junction-gate effect, and both types of carriers accumulate at opposite sides of the material (Fig. 3g). The accumulation of one type of photogenerated carrier between the source and drain leads to a shift in the Fermi level that, if adequately designed, lines up with electrode work functions facilitating charge collection 
under an applied voltage. This was first implemented by Adinolfi et al. in 2015 by employing a softly $n$-doped PbS CQD layer depleted by a deep work function $\mathrm{MoO}_{3}$ layer. ${ }^{88}$ The use of this architecture provides gain while breaking prior photoconductors' response/speed/dark current trade-off. This is achieved by ensuring that, under dark conditions, the channel is fully depleted due to a rectifying junction between the deep-work-function material and the CQD layer. A $10 \mu$ s record rise time for a gain-providing device was achieved with a two-orders-ofmagnitude improvement in photocurrent-to-dark-current ratio compared to photoconductors.

In summary, the use of solution processable materials allows the realization of hybrid phototransistor architectures which have the potential to overcome traditional photodetection limitations posed by the dark current/gain/temporal response compromise. In the next section we review how all of these new solution processable semiconductor photodetectors compare with incumbent technologies, and discuss some new innovations going forward.

\section{Current Status, New Challenges and Novel Approaches}

Detectivity: solution processed semiconductor-based photodetectors compete now with standard elemental inorganic semiconductors (such as Si or Ge) and high-temperature epitaxy semiconductor (such as InGaAs or $\mathrm{HgCdTe}$ ) photodetectors in terms of detectivity (Fig 4a). Now that relevant metrics of performance are on par with these established technologies, the next major barrier stems in their effective incorporation onto standard ROIC arrays. In this sense, interface management is expected to play a crucial role in achieving efficient charge transfer from each pixel. ${ }^{89}$ Compatibility of chemical processing with metal electrodes must be ensured, and this is especially important for halide containing materials such as MHPs. Device stability under operating conditions needs to be further improved to match the standards of this long established industry. Inter-pixel reproducibility is also an important factor to be taken into account in material systems where the grain sizes are comparable with the pixel area (e.g. MHPs). 
In terms of performance, significant improvements can be expected from the hybrid phototransistor platform that, by virtue of breaking of the gain-dark current compromise, offer an unexplored scenario of possibilities. Prominent advances could yet be envisaged by maintaining, or even increasing, the modulation of the transport channel. This is often altered upon the incorporation of the photoactive layers. Interface, together with contact management, is expected to play a significant role in this architecture's near future. The reduction of interface trap states is important to achieve high sub-threshold swings (the amount of voltage required to produce a one decade change in dark current) that increase the SNR. ${ }^{90}$

The growing space in the 2D materials library, as well as the increasing understanding after intense research in the optoelectronic properties photosensitizing layer, picture a promising future for this device architecture.

Charge management within the photosensitizing layer is expected to result in a higher degree of control over carrier injection into the transport phase. This has been recently explored by Nikitskiy and coworkers, who by incorporating a photodiode to actively manipulate carrier injection from the CQD layer into graphene, achieved significant improvements in time response $(\mathrm{BW} \sim 1.5 \mathrm{kHz})$, injection efficiencies (approaching 100\%) and detectivity $\left(D^{*} \sim 10^{13}\right.$ Jones). ${ }^{91}$

A yet open grand challenge is the implementation of efficient mid-IR solution processed sensors, which are of great importance for spectroscopy and aerospace or astronomy imaging applications. Although significant breakthroughs have been reported from material science to devices, ${ }^{92}$ there is still significant room for improvement in order to match, or surpass, the performance of standard $\mathrm{HgCdTe}$ composite photodetectors, currently showing $D^{*}$ on the order of $\sim 10^{10}$ Jones at $10 \mu \mathrm{m}$. Those materials could especially benefit from their integration with hybrid phototransistor architectures. 
Intraband photodetection (1Se-1Pe transition) based HgSe CQDs was demonstrated recently by Deng et al..$^{93}$ A precise doping of the nanocrystals was required in order to populate the $1 \mathrm{Se}$ state, giving rise to photosensitization up to $5 \mu \mathrm{m}$. This new strategy opens the door to colloidal infrared photodetectors that go beyond classic interband sensitization.

If used for aerospace or defense applications, IR photodetectors will need to fulfill stringent performance requirements under harsh conditions, which will open exciting directions of research for the next decade.

Detectivity and speed: along with the requisite for sensitive photodetection there is the increasing demand for fast photodetection, crucial for communication but also for time-offlight imaging. Although several materials and architectures exhibit response times sub-ms (suitable for NSTC/PAL frame rate video standards), the read-out process within the CMOS electronic circuitry continues to rely on speeds fast enough for a full pixel column to be read within a frame time.

A chart comparing $D^{*}$ with $3 \mathrm{~dB}$ bandwidth reveals the scattering between the different material families and architectures (Fig 4b). In this regard, there is a clear difference and an apparent compromise between speed, sensitivity and spectral coverage, as the realization of high quality materials becomes more challenging for lower bandgaps. Organic and organohalide semiconductors exhibit in general higher $B W \times D^{*}$ products for visible and NIR photodetection. ${ }^{67}$ In CQDs, quantum funneling allowed recently achieving $D^{*}$ over $10^{13}$ Jones with remarkable $>1 \mathrm{MHz}$ bandwidths for visible-NIR imaging. In the short-wave IR, this metric has been maximized in CQD photodiodes with $5 \mathrm{MHz}$ bandwidth and $D^{*} \sim 10^{12}$ Jones.

To this point, metal halide perovskites represent the most potential material route for achieving higher $B W \times D^{*}$ products. Sensitization into the IR could be achieved by its synergistic 
combination with other materials such as CQDs in standard or hybrid phototransistor architectures seeking to maintain or increase this metric into the IR.

Gain and bandwidth: these differences also translate into the gain-bandwidth product. CQD photoconductors exhibit the largest gains, but at the expense of moderate bandwidths. Best reported $G \times B W$ products above $6 \cdot 10^{6} \mathrm{~Hz}$ correspond to visible CdS photoconductors of improved carrier mobility. ${ }^{47}$ Organic and organohalide perovskites showcase a better performance in this sense, and organic photodiodes with $B W$ up to $430 \mathrm{MHz}$ (but gain-less) that surpass the $10^{8} \mathrm{~Hz} G \times B W$ barrier were early reported. ${ }^{94}$

This threshold was not surpassed again until the advent of hybrid phototransistors CQDs $\left(\sim 10^{8}\right.$ $\mathrm{Hz})$ and perovskite $\left(\sim 10^{7} \mathrm{~Hz}\right)$ phototransistors,${ }^{79,86}$ fact that showcases the benefit of this hybrid photodetection approach in breaking the $G \times B W$ compromise even for lower bandgap materials. More recently, the rapid progress in MHPs material's processing has led to the realization of methyl-ammonium lead bromide single crystals that, implemented in a back-to-back Schottky photodetector architecture, also resulted in $G \times B W$ beyond the $10^{8} \mathrm{~Hz}$ threshold. ${ }^{95}$ Ultrafast infrared photodetection with moderate quantum efficiencies has been recently reported by Gao et al. by the fabrication of nanosized PbSe microstrip photodetectors. ${ }^{96}$

From a materials perspective, exciting directions are open by the use of recently reported material platforms. Novel hybrid materials such as quantum-dot-in-perovskite solids ${ }^{97}$ - that epitaxially incorporate CQDs in a perovskite matrix - 2D and quasi-2D perovskites ${ }^{98}$ - that exhibit tunable bandgaps and anisotropic mobilities - or perovskite-based nanocrystals, ${ }^{99}$ might open the door for new unconventional photodetection schemes stemming for their particular material properties; or allow efficiently revisiting more traditional approaches employed for crystalline semiconductors such as avalanche ${ }^{100}$ or quantum well photodetectors. ${ }^{21,101}$ The realization of efficient all-solution-processed photodetectors is also an attractive route to be explored towards flexible and wearable photodetection. ${ }^{102}$ 
In the next section we discuss novel functionalities that, enabled by the use of solution processed materials, allow the realization of single pixel multispectral narrowband photodetection as an example of where the technology may take us.

\section{Selective photodetection and charge collection narrowing (CCN)}

Wavelength selective photodetection is a key requirement in order to achieve color discrimination, which is crucial for many applications such as color photography, machine vision, gaming and intelligent surveillance. Current image sensors are based on broadband photojunctions integrated with optical filter arrays in order to attain color selectivity (Fig. 5a). In standard camera sensors, the usage of passive color filter arrays limits the spatial resolution and causes color constancy problem at different illumination conditions. Although passive filters can deliver enough color discrimination in order for reconstruction of standard color photographs, they are not ideal for machine vision and object recognition because their spectral response at different colors strongly overlap.

Stacked image sensors, where individual layers collect different colour channels (Fig. 5b), have also been implemented based on organic semiconductors ${ }^{103-106}$ or CQDs. ${ }^{44}$ This approach, which results in increased sensor resolution, requires a careful design in order to avoid vertical colour-crossover arising from incomplete absorption. Their implementation into top-surface photodetection or back-surface-illuminated architectures demands for the use of transparent thin-film-transistors ${ }^{105}$ that take advantage of the $100 \%$ fill-factor.

The use of single materials for highly colour selective imaging is especially attractive for machine vision, and convenient for ROIC integration (Fig 5c). To this end, narrowband absorbing organic semiconductors have been explored which can potentially satisfy narrowband responses required for machine vision. ${ }^{5}$ However, these devices often use a blend of donor and acceptor (predominantly fullerenes) - both contributing in photocurrent 
generation and need to be optimized in terms of the spectral shape of light absorption. This has been done for the donor part ${ }^{6,107}$ but yet to achieve for the acceptor.

A new approach has been recently introduced that paradoxically uses broadband absorbers to deliver narrowband spectral response. ${ }^{5,108}$ Broadband absorbing polymer:fullerene systems have been employed in optically thick junctions $(1.5-3 \mu \mathrm{m})$ and delivered narrowband spectral responses in red and near-IR with FWHM $<100 \mathrm{~nm}, D^{*}$ exceeding $10^{12}$ Jones and $3 \mathrm{~dB}$ frequency bandwidth of 100's of kHz. ${ }^{5}$ This mechanism has been termed charge collection narrowing $(\mathrm{CCN})$ in which only those charges generated in the volume of the active layer are collected. The idea is based on the fact that the electro-optics of thick junctions $(d>\mu m)$ is substantially different to that of thin photodiodes (d 100's nm). Recent studies further support this concept. ${ }^{109}$ In particular, it has been shown that depending on electron hole mobility ratio, the junction can be electrically thicker by choosing either inverted or conventional architectures. ${ }^{110}$ For a given BHJ system, all photons with energy greater than the optical gap are absorbed near the surface of the transparent anode, whilst those at the optical gap with much lower extinction coefficient can propagate through the cavity. This results in strong absorption and photogeneration in the volume (Fig. 5d). Surface generated carriers must travel back to the cathode resulting in very slow extraction. ${ }^{108}$ This gives rise to the formation space charges accumulating near the anode. Carriers generated within the volume, on the other hand, experience a more balanced electron and hole transport and can be extracted.

$\mathrm{CCN}$ was proposed as a material-agnostic approach. ${ }^{5}$ Its usage has been now expanded to metal halide perovskite films, single crystals and quantum dots. ${ }^{73,111,112}$ Lin et al. ${ }^{73}$ have realized redgreen-blue narrowband photodiodes by introducing ionic dyes in lead iodide and lead perovskites with different halide ratios. The ionic dye reduces the crystallite size and suppresses the mobility. This makes the junction effectively thicker for charge extraction. Simultaneously, it also adjusts the absorption spectrum to the desired shape for CCN (Fig. 5e). 
Following the recent developments in the growth of lead halide perovskite single crystals, Fang et al. developed ultra-narrow band response CCN photodiodes (FWHM $<20 \mathrm{~nm}) .{ }^{112}$ As a result of the low energetic disorder of these single crystals, the absorption onset is sharp and consequently very narrow responses of $<20 \mathrm{~nm}$ can be achieved (Fig. 5f). CCN has also been recently achieved in high gain nanocomposites where spectral narrowing and large gain were simultaneously realized using P3HT:PCBM:CdTe nano-composites. ${ }^{113}$

In sum, color selectivity though charge collection narrowing opens the door for efficient sensing suitable for machine vision applications. Whereas the use of single crystal metal halide perovskites is not appealing for consumer optoelectronics, for it requires mm-thick slabs not compatible with ultrathin compact sensors, the implementation of $\mathrm{CCN}$ based on BHJ or dyemodified perovskites is compatible with CMOS electronics top-surface photodetection architectures ultimately allowing for multispectral sensors that combine different functionalities.

\section{Conclusion}

Photodetection based on solution processed semiconductors has seen tremendous progress during the last decade aided by advances in material science and device engineering. This has led to sensors that combine desirable manufacturing advantages and state-of-the-art performance metrics in a range of applications. The emergence of novel materials such as organohalide perovskites offers further transformative improvements that stem from their exceptional optoelectronic properties. New hybrid phototransistor architectures have also emerged that go beyond the classic photodetection limits. Filter-less, colour selective, multispectral narrowband photodetection is now a real possibility and will allow high purity, more compact image sensors with higher spatial resolutions and pure colour replication. These are very exciting times for photodetection as laboratory ideas become technological realities. 



\section{References}

1. Suzuki, T. Challenges of image-sensor development. in 2010 IEEE International Solid-State Circuits Conference - (ISSCC) 27-30 (IEEE, 2010). doi:10.1109/ISSCC.2010.5434065

2. OmniVision. at http://www.ovt.com/

3. InVisage. (2008). at http://www.invisage.com/

4. Lee, K.-H. et al. Dynamic Characterization of Green-Sensitive Organic Photodetectors Using Nonfullerene Small Molecules: Frequency Response Based on the Molecular Structure. J. Phys. Chem. C 118, 13424-13431 (2014).

5. Armin, A., Jansen-van Vuuren, R. D., Kopidakis, N., Burn, P. L. \& Meredith, P. Narrowband light detection via internal quantum efficiency manipulation of organic photodiodes. Nat. Commun. 6, 6343 (2015).

6. Lyons, D. M. et al. Narrow band green organic photodiodes for imaging. Org. Electron. 15, 2903-2911 (2014).

7. Gong, X. et al. High-detectivity polymer photodetectors with spectral response from $300 \mathrm{~nm}$ to $1450 \mathrm{~nm}$. Science 325, 1665-7 (2009).

8. Armin, A. et al. Thick junction broadband organic photodiodes. Laser Photon. Rev. 8, 924-932 (2014).

9. Konstantatos, G. et al. Ultrasensitive solution-cast quantum dot photodetectors. Nature 442, 180-3 (2006).

10. Clifford, J. P. et al. Fast, sensitive and spectrally tuneable colloidal-quantum-dot photodetectors. Nat. Nanotechnol. 4, 40-4 (2009).

11. Saran, R. \& Curry, R. J. Lead sulphide nanocrystal photodetector technologies. Nat. Photonics 10, 81-92 (2016).

12. Mohd Yusoff, A. R. Bin \& Nazeeruddin, M. K. Organohalide Lead Perovskites for Photovoltaic Applications. J. Phys. Chem. Lett. 7, 851-866 (2016).

13. Brenner, T. M., Egger, D. A., Kronik, L., Hodes, G. \& Cahen, D. Hybrid organicinorganic perovskites: low-cost semiconductors with intriguing charge-transport properties. Nat. Rev. Mater. 1, 15007 (2016). 
14. Sze, S. M. Physics of Semiconductor Devices. (John Wiley \& Sons, 1981).

15. Gong, X. et al. High-detectivity polymer photodetectors with spectral response from $300 \mathrm{~nm}$ to $1450 \mathrm{~nm}$. Science 325, 1665-7 (2009).

16. Liu, C. et al. Ultrasensitive solution-processed broad-band photodetectors using $\mathrm{CH}_{3} \mathrm{NH}_{3} \mathrm{PbI}_{3}$ perovskite hybrids and $\mathrm{PbS}$ quantum dots as light harvesters. Nanoscale 7, 16460-9 (2015).

17. Gong, X. et al. Semiconducting polymer photodetectors with electron and hole blocking layers: high detectivity in the near-infrared. Sensors (Basel). 10, 6488-96 (2010).

18. Liu, X., Wang, H., Yang, T., Zhang, W. \& Gong, X. Solution-processed ultrasensitive polymer photodetectors with high external quantum efficiency and detectivity. ACS Appl. Mater. Interfaces 4, 3701-5 (2012).

19. Hu, X. et al. High-detectivity inverted near-infrared polymer photodetectors using cross-linkable conjugated polyfluorene as an electron extraction layer. J. Mater. Chem. C 2, 9592-9598 (2014).

20. Liu, C. et al. Ultrasensitive solution-processed perovskite hybrid photodetectors. $J$. Mater. Chem. C 3, 6600-6606 (2015).

21. Rogalski, A. Infrared Detectors. CRC Press (2010).

22. Liu, H., Lhuillier, E. \& Guyot-Sionnest, P. 1/f noise in semiconductor and metal nanocrystal solids. J. Appl. Phys. 115, 154309 (2014).

23. McNeill, R., Siudak, R., Wardlaw, J. \& Weiss, D. Electronic Conduction in Polymers. I. The Chemical Structure of Polypyrrole. Aust. J. Chem. 16, 1056 (1963).

24. Tang, C. W. Photovoltaic effects of metal-chlorophyll-a-metal sandwich cells. $J$. Chem. Phys. 62, 2139 (1975).

25. Koezuka, H., Tsumura, A. \& Ando, T. Field-effect transistor with polythiophene thin film. Synth. Met. 18, 699-704 (1987).

26. Baeg, K.-J., Binda, M., Natali, D., Caironi, M. \& Noh, Y.-Y. Organic light detectors: photodiodes and phototransistors. Adv. Mater. 25, 4267-95 (2013).

27. Harrison, M. G., Grüner, J. \& Spencer, G. C. W. Analysis of the photocurrent action spectra of MEH-PPV polymer photodiodes. Phys. Rev. B 55, 7831-7849 (1997). 
28. Armin, A. et al. Spectral dependence of the internal quantum efficiency of organic solar cells: effect of charge generation pathways. J. Am. Chem. Soc. 136, 11465-72 (2014).

29. Fang, Y., Guo, F., Xiao, Z. \& Huang, J. Large Gain, Low Noise Nanocomposite Ultraviolet Photodetectors with a Linear Dynamic Range of $120 \mathrm{~dB}$. Adv. Opt. Mater. 2, 348-353 (2014).

30. Li, L., Huang, Y., Peng, J., Cao, Y. \& Peng, X. Highly responsive organic nearinfrared photodetectors based on a porphyrin small molecule. J. Mater. Chem. C 2, $1372(2014)$.

31. Yao, Y. et al. Plastic Near-Infrared Photodetectors Utilizing Low Band Gap Polymer. Adv. Mater. 19, 3979-3983 (2007).

32. Pierre, A., Deckman, I., Lechêne, P. B. \& Arias, A. C. High Detectivity All-Printed Organic Photodiodes. Adv. Mater. n/a-n/a (2015). doi:10.1002/adma.201502238

33. You, J. et al. A polymer tandem solar cell with $10.6 \%$ power conversion efficiency. Nat. Commun. 4, 1446 (2013).

34. Coffin, R. C., Peet, J., Rogers, J. \& Bazan, G. C. Streamlined microwave-assisted preparation of narrow-bandgap conjugated polymers for high-performance bulk heterojunction solar cells. Nat. Chem. 1, 657-61 (2009).

35. Armin, A. et al. Quantum Efficiency of Organic Solar Cells: Electro-Optical Cavity Considerations. ACS Photonics 1, 173-181 (2014).

36. Lupton, J. M. et al. Organic Microcavity Photodiodes. Adv. Mater. 15, 1471-1474 (2003).

37. Qi, J. et al. Panchromatic small molecules for UV-Vis-NIR photodetectors with high detectivity. J. Mater. Chem. C 2, 2431 (2014).

38. Rauch, T. et al. Near-infrared imaging with quantum-dot-sensitized organic photodiodes. Nat. Photonics 3, 332-336 (2009).

39. Guo, F. et al. A nanocomposite ultraviolet photodetector based on interfacial trapcontrolled charge injection. Nat. Nanotechnol. 7, 798-802 (2012).

40. Dong, R. et al. An Ultraviolet-to-NIR Broad Spectral Nanocomposite Photodetector with Gain. Adv. Opt. Mater. 2, 549-554 (2014). 
41. Greenham, N. C., Peng, X. \& Alivisatos, A. P. Charge separation and transport in conjugated-polymer/semiconductor-nanocrystal composites studied by photoluminescence quenching and photoconductivity. Phys. Rev. B 54, 17628-17637 (1996).

42. Oertel, D. C., Bawendi, M. G., Arango, A. C. \& Bulović, V. Photodetectors based on treated CdSe quantum-dot films. Appl. Phys. Lett. 87, 213505 (2005).

43. McDonald, S. a et al. Solution-processed $\mathrm{PbS}$ quantum dot infrared photodetectors and photovoltaics. Nat. Mater. 4, 138-42 (2005).

44. Konstantatos, G., Clifford, J., Levina, L. \& Sargent, E. H. Sensitive solution-processed visible-wavelength photodetectors. Nat. Photonics 1, 531-534 (2007).

45. Konstantatos, G. \& Sargent, E. H. PbS colloidal quantum dot photoconductive photodetectors: Transport, traps, and gain. Appl. Phys. Lett. 91, 173505 (2007).

46. Konstantatos, G., Levina, L., Fischer, A. \& Sargent, E. H. Engineering the temporal response of photoconductive photodetectors via selective introduction of surface trap states. Nano Lett. 8, 1446-50 (2008).

47. Lee, J.-S., Kovalenko, M. V, Huang, J., Chung, D. S. \& Talapin, D. V. Band-like transport, high electron mobility and high photoconductivity in all-inorganic nanocrystal arrays. Nat. Nanotechnol. 6, 348-52 (2011).

48. Kim, S. J., Kim, W. J., Sahoo, Y., Cartwright, A. N. \& Prasad, P. N. Multiple exciton generation and electrical extraction from a PbSe quantum dot photoconductor. Appl. Phys. Lett. 92, 031107 (2008).

49. Sukhovatkin, V., Hinds, S., Brzozowski, L. \& Sargent, E. H. Colloidal quantum-dot photodetectors exploiting multiexciton generation. Science 324, 1542-4 (2009).

50. Ka, I. et al. Multiple exciton generation induced enhancement of the photoresponse of pulsed-laser-ablation synthesized single-wall-carbon-nanotube/PbS-quantum-dots nanohybrids. Sci. Rep. 6, 20083 (2016).

51. Gao, J., Fidler, A. F. \& Klimov, V. I. Carrier multiplication detected through transient photocurrent in device-grade films of lead selenide quantum dots. Nat. Commun. $\mathbf{6}$, 8185 (2015).

52. Nair, G., Geyer, S. M., Chang, L.-Y. \& Bawendi, M. G. Carrier multiplication yields 
in $\mathrm{PbS}$ and $\mathrm{PbSe}$ nanocrystals measured by transient photoluminescence. Phys. Rev. $B$ 78, 125325 (2008).

53. Clifford, J. P., Johnston, K. W., Levina, L. \& Sargent, E. H. Schottky barriers to colloidal quantum dot films. Appl. Phys. Lett. 91, 253117 (2007).

54. Pal, B. N. et al. High-Sensitivity p-n Junction Photodiodes Based on PbS Nanocrystal Quantum Dots. Adv. Funct. Mater. 22, 1741-1748 (2012).

55. Szendrei, K. et al. Solution-Processable Near-IR Photodetectors Based on Electron Transfer from PbS Nanocrystals to Fullerene Derivatives. Adv. Mater. 21, 683-687 (2009).

56. Kim, J. Y. et al. Single-step fabrication of quantum funnels via centrifugal colloidal casting of nanoparticle films. Nat. Commun. 6, 7772 (2015).

57. García de Arquer, F. P., Beck, F. J., Bernechea, M. \& Konstantatos, G. Plasmonic light trapping leads to responsivity increase in colloidal quantum dot photodetectors. Appl. Phys. Lett. 100, 043101 (2012).

58. Beck, F. J., Stavrinadis, A., Diedenhofen, S. L., Lasanta, T. \& Konstantatos, G. Surface Plasmon Polariton Couplers for Light Trapping in Thin-Film Absorbers and Their Application to Colloidal Quantum Dot Optoelectronics. ACS Photonics 141007152154008 (2014). doi:10.1021/ph5002704

59. Diedenhofen, S. L., Kufer, D., Lasanta, T. \& Konstantatos, G. Integrated colloidal quantum dot photodetectors with color-tunable plasmonic nanofocusing lenses. Light Sci. Appl. 4, e234 (2015).

60. Beck, F. J., Garcia de Arquer, F. P., Bernechea, M. \& Konstantatos, G. Electrical effects of metal nanoparticles embedded in ultra-thin colloidal quantum dot films. Appl. Phys. Lett. 101, 041103 (2012).

61. García de Arquer, F. P., Lasanta, T., Bernechea, M. \& Konstantatos, G. Tailoring the Electronic Properties of Colloidal Quantum Dots in Metal-Semiconductor Nanocomposites for High Performance Photodetectors. Small 11, 2636-41 (2015).

62. Lin, Q., Armin, A., Nagiri, R. C. R., Burn, P. L. \& Meredith, P. Electro-optics of perovskite solar cells. Nat. Photonics 9, 106-112 (2014).

63. Kojima, A., Teshima, K., Shirai, Y. \& Miyasaka, T. Organometal halide perovskites as 
visible-light sensitizers for photovoltaic cells. J. Am. Chem. Soc. 131, 6050-1 (2009).

64. Lee, M. M., Teuscher, J., Miyasaka, T., Murakami, T. N. \& Snaith, H. J. Efficient hybrid solar cells based on meso-superstructured organometal halide perovskites. Science 338, 643-7 (2012).

65. $\mathrm{Hu}, \mathrm{X}$. et al. High-Performance Flexible Broadband Photodetector Based on Organolead Halide Perovskite. Adv. Funct. Mater. 24, 7373-7380 (2014).

66. Lin, Q., Armin, A., Lyons, D. M., Burn, P. L. \& Meredith, P. Low noise, IR-blind organohalide perovskite photodiodes for visible light detection and imaging. $A d v$. Mater. 27, 2060-4 (2015).

67. Dou, L. et al. Solution-processed hybrid perovskite photodetectors with high detectivity. Nat. Commun. 5, 5404 (2014).

68. Sutherland, B. R. et al. Sensitive, Fast, and Stable Perovskite Photodetectors Exploiting Interface Engineering. ACS Photonics 2, 1117-1123 (2015).

69. Fang, Y. \& Huang, J. Resolving Weak Light of Sub-picowatt per Square Centimeter by Hybrid Perovskite Photodetectors Enabled by Noise Reduction. Adv. Mater. 27, 2804-10 (2015).

70. Liu, M., Johnston, M. B. \& Snaith, H. J. Efficient planar heterojunction perovskite solar cells by vapour deposition. Nature 501, 395-8 (2013).

71. Jeon, N. J. et al. Solvent engineering for high-performance inorganic-organic hybrid perovskite solar cells. Nat. Mater. 13, 897-903 (2014).

72. Dong, R. et al. High-gain and low-driving-voltage photodetectors based on organolead triiodide perovskites. Adv. Mater. 27, 1912-8 (2015).

73. Lin, Q., Armin, A., Burn, P. L. \& Meredith, P. Filterless narrowband visible photodetectors. Nat. Photonics 9, 687-694 (2015).

74. Yakunin, S. et al. Detection of X-ray photons by solution-processed lead halide perovskites. Nat. Photonics 9, 444-449 (2015).

75. Wei, H. et al. Sensitive X-ray detectors made of methylammonium lead tribromide perovskite single crystals. Nat. Photonics 10, 333-339 (2016).

76. Shah, K. . et al. Lead iodide X-ray detection systems. Nucl. Instruments Methods Phys. Res. Sect. A Accel. Spectrometers, Detect. Assoc. Equip. 380, 266-270 (1996). 
77. Wei, H. et al. Sensitive X-ray detectors made of methylammonium lead tribromide perovskite single crystals. Nat. Photonics 10, 333-339 (2016).

78. Gu, P., Yao, Y., Feng, L., Niu, S. \& Dong, H. Recent advances in polymer phototransistors. Polym. Chem. 6, 7933-7944 (2015).

79. Konstantatos, G. et al. Hybrid graphene-quantum dot phototransistors with ultrahigh gain. Nat. Nanotechnol. 7, 363-8 (2012).

80. Sun, Z. et al. Infrared photodetectors based on CVD-grown graphene and $\mathrm{PbS}$ quantum dots with ultrahigh responsivity. Adv. Mater. 24, 5878-83 (2012).

81. Wang, Y. et al. Hybrid Graphene-Perovskite Phototransistors with Ultrahigh Responsivity and Gain. Adv. Opt. Mater. 3, 1389-1396 (2015).

82. Lee, Y. et al. High-Performance Perovskite-Graphene Hybrid Photodetector. Adv. Mater. 27, 41-46 (2015).

83. Li, F. et al. Ambipolar solution-processed hybrid perovskite phototransistors. Nat. Commun. 6, 8238 (2015).

84. Kufer, D. et al. Hybrid 2D-0D MoS2 -PbS quantum dot photodetectors. Adv. Mater. 27, 176-80 (2015).

85. Kufer, D. \& Konstantatos, G. Highly Sensitive, Encapsulated MoS2 Photodetector with Gate Controllable Gain and Speed. Nano Lett. 15, 7307-13 (2015).

86. Wang, H. et al. Achieving ultrahigh carrier mobility and photo-responsivity in solution-processed perovskite/carbon nanotubes phototransistors. (2015). at http://arxiv.org/abs/1512.03893

87. Yuan, Y. et al. Solution-processed nanoparticle super-float-gated organic field-effect transistor as un-cooled ultraviolet and infrared photon counter. Sci. Rep. 3, 2707 (2013).

88. Adinolfi, V. et al. Photojunction field-effect transistor based on a colloidal quantum dot absorber channel layer. ACS Nano 9, 356-62 (2015).

89. Masala, S. et al. The Silicon:Colloidal Quantum Dot Heterojunction. Adv. Mater. 27, 7445-7450 (2015).

90. Kufer, D., Lasanta, T., Bernechea, M., Koppens, F. H. L. \& Konstantatos, G. Interface Engineering in Hybrid Quantum Dot-2D Phototransistors. (2016). 
91. Nikitskiy, I. et al. Integrating an electrically active colloidal quantum dot photodiode with a graphene phototransistor. Nat. Commun. 7, 11954 (2016).

92. Keuleyan, S., Lhuillier, E., Brajuskovic, V. \& Guyot-Sionnest, P. Mid-infrared HgTe colloidal quantum dot photodetectors. Nat. Photonics 5, 489-493 (2011).

93. Deng, Z., Jeong, K. S. \& Guyot-Sionnest, P. Colloidal Quantum Dots Intraband Photodetectors. (2014).

94. Peumans, P., Bulović, V. \& Forrest, S. R. Efficient, high-bandwidth organic multilayer photodetectors. Appl. Phys. Lett. 76, 3855 (2000).

95. Saidaminov, M. I. et al. Planar-integrated single-crystalline perovskite photodetectors. Nat. Commun. 6, 8724 (2015).

96. Gao, J., Nguyen, S. C., Bronstein, N. D. \& Alivisatos, A. P. Solution-Processed, HighSpeed, and High-Quantum-Efficiency Quantum Dot Infrared Photodetectors. (2016).

97. Ning, Z. et al. Quantum-dot-in-perovskite solids. Nature 523, 324-328 (2015).

98. Saparov, B. \& Mitzi, D. B. Organic-Inorganic Perovskites: Structural Versatility for Functional Materials Design. Chem. Rev. 116, 4558-4596 (2016).

99. Ramasamy, P. et al. All-inorganic cesium lead halide perovskite nanocrystals for photodetector applications. Chem. Commun. (Camb). 52, 2067-70 (2016).

100. Hayden, O., Agarwal, R. \& Lieber, C. M. Nanoscale avalanche photodiodes for highly sensitive and spatially resolved photon detection. Nat. Mater. 5, 352-6 (2006).

101. Martyniuk, P. \& Rogalski, A. Quantum-dot infrared photodetectors: Status and outlook. Prog. Quantum Electron. 32, 89-120 (2008).

102. Choi, J.-H. et al. Exploiting the colloidal nanocrystal library to construct electronic devices. Science 352, 205-208 (2016).

103. Aihara, S. et al. Stacked Image Sensor With Green- and Red-Sensitive Organic Photoconductive Films Applying Zinc Oxide Thin-Film Transistors to a Signal Readout Circuit. IEEE Trans. Electron Devices 56, 2570-2576 (2009).

104. Antognazza, M. R., Scherf, U., Monti, P. \& Lanzani, G. Organic-based tristimuli colorimeter. Appl. Phys. Lett. 90, 163509 (2007).

105. Seo, H. et al. A 128×96 Pixel Stack-Type Color Image Sensor: Stack of Individual 
Blue-, Green-, and Red-Sensitive Organic Photoconductive Films Integrated with a ZnO Thin Film Transistor Readout Circuit. Jpn. J. Appl. Phys. 50, 024103 (2011).

106. Lim, S.-J. et al. Organic-on-silicon complementary metal-oxide-semiconductor colour image sensors. Sci. Rep. 5, 7708 (2015).

107. Jansen-van Vuuren, R. D., Pivrikas, A., Pandey, A. K. \& Burn, P. L. Colour selective organic photodetectors utilizing ketocyanine-cored dendrimers. J. Mater. Chem. C 1, $3532(2013)$.

108. Johnston, M. B. Optoelectronics: Colour-selective photodiodes. Nat. Photonics 9, 634$636(2015)$.

109. Tait, J. G. et al. Interfacial Depletion Regions: Beyond the Space Charge Limit in Thick Bulk Heterojunctions. ACS Appl. Mater. Interfaces 8, 2211-9 (2016).

110. Armin, A. et al. Electro-Optics of Conventional and Inverted Thick Junction Organic Solar Cells. ACS Photonics 2, 1745-1754 (2015).

111. Qiao, K. et al. Spectra-selective PbS quantum dot infrared photodetectors. Nanoscale 8, 7137-43 (2016).

112. Fang, Y., Dong, Q., Shao, Y., Yuan, Y. \& Huang, J. Highly narrowband perovskite single-crystal photodetectors enabled by surface-charge recombination. Nat. Photonics 9, 679-686 (2015).

113. Shen, L., Fang, Y., Wei, H., Yuan, Y. \& Huang, J. A Highly Sensitive Narrowband Nanocomposite Photodetector with Gain. Adv. Mater. 28, 2043-8 (2016).

114. Yu, G., Wang, J., McElvain, J. \& Heeger, A. J. Large-Area, Full-Color Image Sensors Made with Semiconducting Polymers. Adv. Mater. 10, 1431-1434 (1998).

115. Hamilton, M. C., Martin, S. \& Kanicki, J. Thin-Film Organic Polymer Phototransistors. IEEE Trans. Electron Devices 51, 877-885 (2004).

116. Agostinelli, T. et al. A polymer/fullerene based photodetector with extremely low dark current for x-ray medical imaging applications. Appl. Phys. Lett. 93, 203305 (2008).

117. Guo, F., Xiao, Z. \& Huang, J. Fullerene Photodetectors with a Linear Dynamic Range of 90 dB Enabled by a Cross-Linkable Buffer Layer. Adv. Opt. Mater. 1, 289-294 (2013).

118. Zhang, H. et al. Transparent organic photodetector using a near-infrared absorbing 
cyanine dye. Sci. Rep. 5, 9439 (2015).

119. Fang, Y. \& Huang, J. Resolving Weak Light of Sub-picowatt per Square Centimeter by Hybrid Perovskite Photodetectors Enabled by Noise Reduction. Adv. Mater. 27, 2804-10 (2015).

120. Solis-Ibarra, D., Smith, I. C. \& Karunadasa, H. I. Post-synthetic halide conversion and selective halogen capture in hybrid perovskites. Chem. Sci. 6, 4054-4059 (2015).

121. Filip, M. R., Eperon, G. E., Snaith, H. J. \& Giustino, F. Steric engineering of metalhalide perovskites with tunable optical band gaps. Nat. Commun. 5, 5757 (2014).

122. Chen, H.-W. et al. A Switchable High-Sensitivity Photodetecting and Photovoltaic Device with Perovskite Absorber. (2015).

123. An, K. H., O’Connor, B., Pipe, K. P. \& Shtein, M. Organic photodetector with spectral response tunable across the visible spectrum by means of internal optical microcavity. Org. Electron. 10, 1152-1157 (2009). 

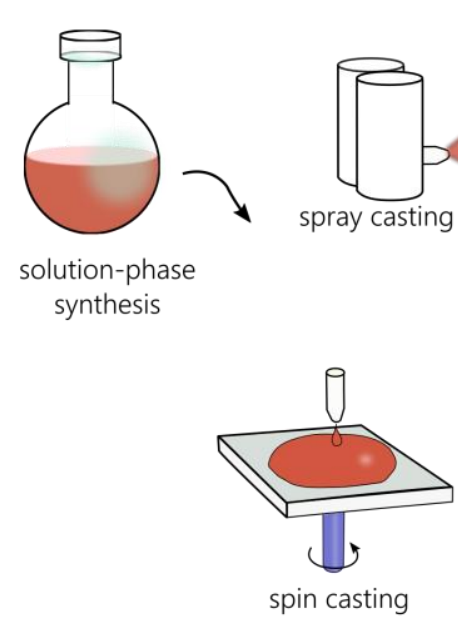

e
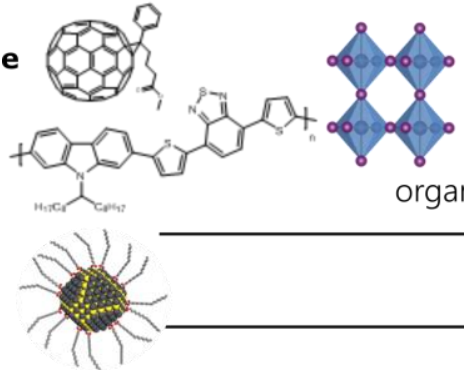

organics \& perovskites
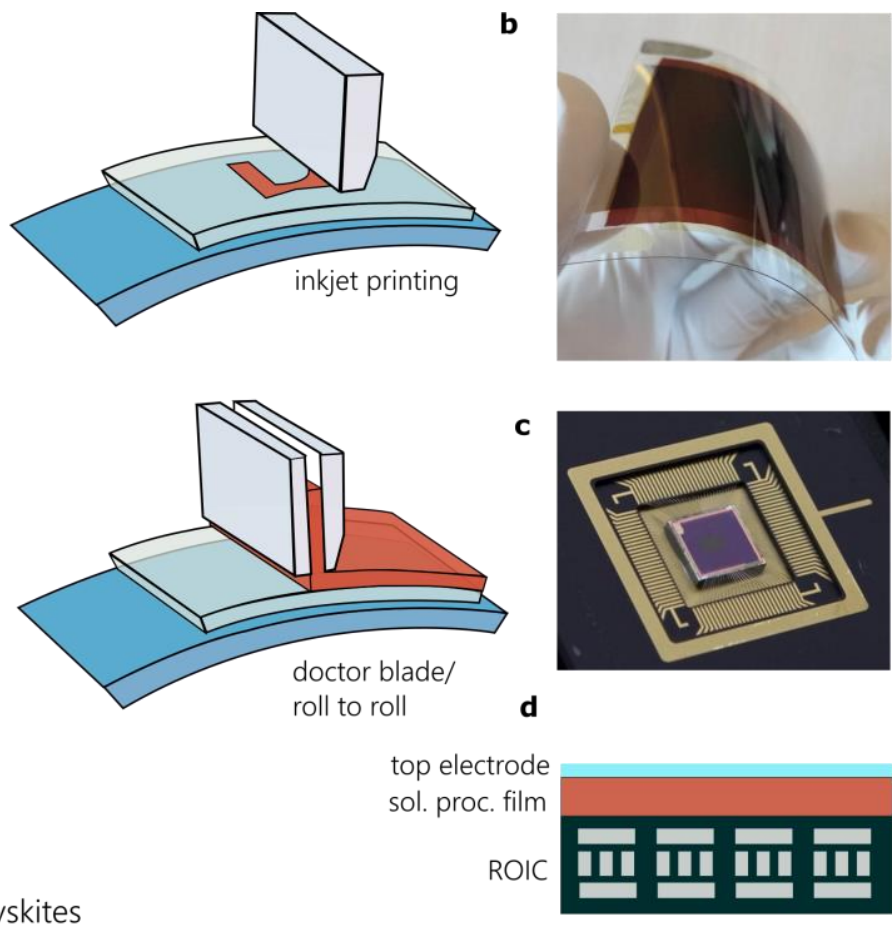

doctor blade roll to roll

d

top electrode sol. proc. film

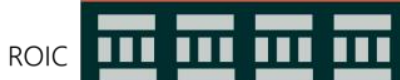

\section{quantum dots}

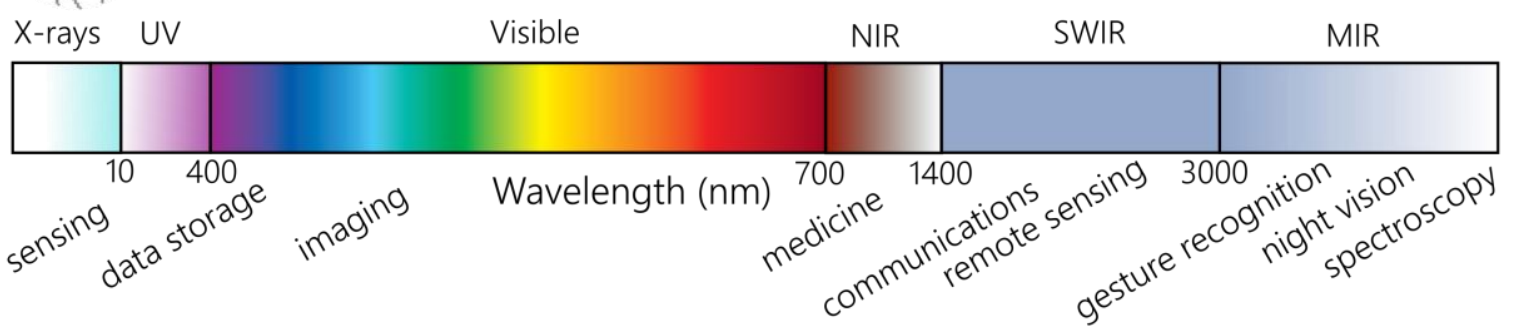

Figure 1 | Solution processed photodetection. (a) Solution processed materials are synthesized resulting in colloidal semiconductor inks. These can be deposited and assembled in solid films with different techniques, such as spray casting or spin coating, or by using already available manufacturing techniques such as inkjet printing, doctor blading or roll-toroll. (b) These methods and materials are compatible with a variety of substrates, flexible and unconventional, especially attractive for example for wearable electronics, but also ensuring compatibility with current sensing technologies such as complementary-metal-oxidesemiconductors (CMOS). This ease of integration has led to novel photodetection schemes, such as top-surface photodetectors (c), where the photoactive material is readily formed on top of the read-out-circuit with total coverage, resulting in improved sensitivity and minimized cross-talk (d). Typical solution processed materials comprise organic semiconductors and dyes, organohalide perovskites and inorganic nanocrystals and quantum dot materials, with a wide spectral range cover applications from X-ray to the mid infrared. 
Table 1 | Photodetector metrics of performance

\begin{tabular}{|c|c|c|}
\hline Metric & Units & Definition \\
\hline Photocurrent $\left(\mathrm{I}_{\mathrm{ph}}\right)$ & A & $\begin{array}{l}\text { Current flowing through a device due to } \\
\text { illumination }\end{array}$ \\
\hline Dark-Current $\left(\mathrm{I}_{\mathrm{d}}\right)$ & A & Current flowing in the absence of illumination \\
\hline Responsivity $(R)$ & $\mathrm{A} / \mathrm{W}(\mathrm{V} / \mathrm{W})$ & $\begin{array}{l}\text { Ratio of the photocurrent to the incident optical } \\
\text { power }\end{array}$ \\
\hline $\begin{array}{l}\text { External Quantum } \\
\text { Efficiency (EQE) }\end{array}$ & $\%$ & $\begin{array}{l}\text { Number of carriers circulating by number of } \\
\text { incident photons }\end{array}$ \\
\hline Gain $(G)$ & unitless & Number of collected carriers by photons absorbed \\
\hline 3dB-bandwidth $(B W)$ & $\mathrm{Hz}$ & $\begin{array}{l}\text { Modulation frequency at which the responsivity } \\
\text { of the device is half of that at steady state } \\
\text { conditions. }\end{array}$ \\
\hline $\begin{array}{l}\text { Linear dynamic range } \\
(L D R)\end{array}$ & $\mathrm{dB}$ & $\begin{array}{l}\text { Range of incident optical powers where the } \\
\text { detector responds linearly }\end{array}$ \\
\hline $\begin{array}{l}\text { Noise spectral density } \\
\left(S_{\mathrm{N}}\right)\end{array}$ & $\mathrm{A} / \mathrm{Hz}^{1 / 2}$ & rms of current fluctuation \\
\hline $\begin{array}{l}\text { Noise Equivalent } \\
\text { Power (NEP) }\end{array}$ & $\mathrm{W} / \mathrm{Hz}^{1 / 2}$ & $\begin{array}{l}\text { Optical power that would be required to generate } \\
S_{\mathrm{N}} \text { It is a measure of the sensitivity of the device } \\
\left(\mathrm{NEP}=S_{\mathrm{N}} / R\right) \text {. }\end{array}$ \\
\hline $\begin{array}{l}\text { Normalized detectivity } \\
\left(D^{*}\right)\end{array}$ & $\begin{array}{c}\mathrm{cm}^{*} \mathrm{~Hz}^{1 / 2} / \mathrm{W} \\
(\text { Jones })^{\dagger}\end{array}$ & $\begin{array}{l}\text { NEP normalized to the area of the device }\left(A_{\mathrm{d}}\right) \\
\text { and electrical bandwidth of the noise } \\
\text { measurement }(B) \text {. } \\
D^{*}=\left(\mathrm{A}_{\mathrm{d}} B\right)^{1 / 2} / \mathrm{NEP}=R\left(A_{\mathrm{d}} B\right)^{1 / 2} / S_{\mathrm{N}}\end{array}$ \\
\hline $\begin{array}{l}\text { Spectral selectivity } \\
\text { (FWHM) }\end{array}$ & $\mathrm{nm}$ & Full-width at half maximum resonance width \\
\hline
\end{tabular}


$\dagger$ For clarity, we will refer to Jones instead as an equivalent to $\mathrm{cm}^{*} \mathrm{~Hz}^{1 / 2} / \mathrm{W}$ throughout the manuscript. 
Table 2 | Figures of merit and progress of solution processed photodetectors

\begin{tabular}{|c|c|c|c|c|c|c|c|c|c|c|}
\hline Year & Ref & Reg. & $\mathrm{EQE} / R / G$ & $\begin{array}{c}\text { D* (Jones) } \\
\text { or NEP } \\
\text { (measured) }\end{array}$ & $\begin{array}{l}\text { D* (Jones) } \\
\text { (inferred) } \dagger\end{array}$ & BW & LDR & Type & Material & Features \\
\hline 1996 & 41 & VIS & $0.2 \mathrm{~A} / \mathrm{W}$ & - & - & - & - & $\mathrm{PC}$ & $\begin{array}{l}\mathrm{CQD} \\
(\mathrm{CdX})\end{array}$ & $\begin{array}{l}\text { Early CQD } \\
\text { photodetector }\end{array}$ \\
\hline 1998 & 114 & $\begin{array}{l}\text { UV- } \\
\text { VIS }\end{array}$ & $0.2 \mathrm{~A} / \mathrm{W}$ & & & & & PD & Polymer & $\begin{array}{l}\text { Image sensor with } \\
\text { color filter }\end{array}$ \\
\hline 2003 & 36 & VIS & $0.2 \mathrm{~mA} / \mathrm{W}$ & - & - & - & - & PD & Polymer & $\begin{array}{l}\text { Color selectivity } \\
\text { using microcavity }\end{array}$ \\
\hline 2004 & 115 & $\begin{array}{c}\text { UV- } \\
\text { Green }\end{array}$ & $0.7 \cdot \mathrm{mA} / \mathrm{W}$ & - & - & - & - & PT & Polymer & $\begin{array}{l}\text { Solution processed } \\
\text { phototransistor }\end{array}$ \\
\hline 2005 & 42 & VIS & EQE $15 \%$ & - & $10^{8}$ & $50 \mathrm{kHz}$ & - & $\mathrm{PC}$ & $\begin{array}{l}\text { CQDs } \\
(\mathrm{CdSe})\end{array}$ & - \\
\hline 2006 & 9 & $\begin{array}{l}\text { VIS- } \\
1.6 u m\end{array}$ & $2700 \mathrm{~A} / \mathrm{W}$ & $2 \cdot 10^{13}$ & $7 \cdot 10^{12}$ & $20 \mathrm{~Hz}$ & - & $\mathrm{PC}$ & $\begin{array}{l}\text { CQDs } \\
(\mathrm{PbS})\end{array}$ & $\begin{array}{l}\text { Pure CQDs. } \\
\text { Ultrasensitive and } \\
\text { high gain }\end{array}$ \\
\hline 2007 & 44 & $\begin{array}{l}\text { VIS- } \\
\text { NIR }\end{array}$ & $120 \mathrm{~A} / \mathrm{W}$ & $5 \cdot 10^{12}$ & $3 \cdot 10^{13}$ & $20 \mathrm{~Hz}$ & $150 \mathrm{~dB}$ & PT & $\begin{array}{l}\text { CQDs } \\
(\mathrm{PbS})\end{array}$ & $\begin{array}{l}\text { Exploiting CQDs } \\
\text { tunability for VIS }\end{array}$ \\
\hline 2007 & 31 & NIR & $0.6 \mathrm{~A} / \mathrm{W}$ & $\begin{array}{c}\text { NEP } \\
1.35 \cdot 10^{-11}\end{array}$ & - & $4 \mathrm{MHz}$ & - & PD & Polymer & $\begin{array}{l}\text { NIR response } \\
\text { using polymers }\end{array}$ \\
\hline 2008 & 116 & X-ray & $5 \mathrm{~mA} / \mathrm{W}$ & $\begin{array}{c}\text { X-Ray } \\
\text { sensitivity } \\
7 \mathrm{mGy} / \mathrm{s}\end{array}$ & - & - & - & $\mathrm{PD}$ & $\begin{array}{l}\text { Polymer } \\
\text { /Fullere } \\
\text { ne }\end{array}$ & $\begin{array}{l}\text { Low noise } \\
\text { enabling X-ray } \\
\text { detection }\end{array}$ \\
\hline 2009 & 15 & $\begin{array}{l}\text { VIS- } \\
\text { NIR }\end{array}$ & $0.2 \mathrm{~A} / \mathrm{W}$ & - & $1 \cdot 10^{12}$ & & $100 \mathrm{~dB}$ & PD & Polymer & NIR sensitivity \\
\hline 2009 & 49 & $\begin{array}{l}\text { VIS- } \\
\text { NIR }\end{array}$ & $G \sim 85$ & - & & $30 \mathrm{~Hz}$ & - & $\mathrm{PC}$ & $\begin{array}{l}\text { CQDs } \\
(\mathrm{PbS})\end{array}$ & Exploiting MEG \\
\hline 2009 & 10 & $\begin{array}{l}\text { VIS- } \\
1.6 \mu \mathrm{m}\end{array}$ & EQE $50 \%$ & $10^{12}$ & & $5 \mathrm{MHz}$ & - & PD & $\begin{array}{l}\text { CQDs } \\
(\mathrm{PbS})\end{array}$ & Ultrafast \\
\hline 2011 & 47 & VIS & $2.5 \mathrm{~A} / \mathrm{W}$ & $10^{13}$ & & $0.4 \mathrm{kHz}$ & & $\mathrm{PC}$ & $\begin{array}{c}\text { CQDs } \\
(\mathrm{CdS}) / \mathrm{C} \\
\mathrm{sSe})\end{array}$ & High mobility \\
\hline
\end{tabular}




\begin{tabular}{|c|c|c|c|c|c|c|c|c|c|c|}
\hline 2012 & 39 & UV & $1000 \mathrm{~A} / \mathrm{W}$ & $7 \cdot 10^{15}$ & & $600 \mathrm{~Hz}$ & - & $\begin{array}{c}\mathrm{PD} / \mathrm{P} \\
\mathrm{C}\end{array}$ & $\begin{array}{c}\mathrm{NCs} \\
(\mathrm{ZnO} / \mathrm{P} \\
\mathrm{VKT})\end{array}$ & $\begin{array}{l}\text { Photodiode/conduc } \\
\text { tor switch }\end{array}$ \\
\hline 2012 & 79 & $\begin{array}{l}\text { VIS- } \\
1.6 u m\end{array}$ & $\begin{array}{l}10^{7} \mathrm{~A} / \mathrm{W} \\
G=10^{8}\end{array}$ & $7 \cdot 10^{13}$ & & $30 \mathrm{~Hz}$ & - & PT & $\begin{array}{l}\text { CQDs } \\
(\mathrm{PbS}-\mathrm{g})\end{array}$ & $\begin{array}{l}\text { Hybrid } \\
\text { phototransistor. } \\
\text { Ultrahigh gain }\end{array}$ \\
\hline 2013 & 117 & $\begin{array}{l}\text { UV- } \\
\text { VIS }\end{array}$ & $0.2 \mathrm{~A} / \mathrm{W}$ & $4 \cdot 10^{11}$ & - & $20 \mathrm{kHz}$ & $180 \mathrm{~dB}$ & PD & $\begin{array}{c}\text { Fulleren } \\
\mathrm{e}\end{array}$ & Large LDR \\
\hline 2014 & 8 & VIS & $0.33 \mathrm{~A} / \mathrm{W}$ & $10^{13}$ & - & $\begin{array}{l}200 \mathrm{kHz} \\
-2 \mathrm{MHz}\end{array}$ & $180 \mathrm{~dB}$ & PD & Polymer & $\begin{array}{l}\text { Large LDR, Low } \\
\text { noise, spectrally } \\
\text { flat, large area }\end{array}$ \\
\hline 2014 & 84 & $\begin{array}{l}\text { VIS- } \\
1.6 u m\end{array}$ & $6 \cdot 10^{5} \mathrm{~A} / \mathrm{W}$ & $5 \cdot 10^{11}$ & $10^{15}$ & $10 \mathrm{~Hz}$ & - & PT & $\begin{array}{l}\mathrm{CQD}+ \\
\mathrm{MoS}_{2}\end{array}$ & $\begin{array}{l}\text { High gain } \\
\text { combined with } \\
\text { channel dark }\end{array}$ \\
\hline 2014 & 65 & UV & $3 \mathrm{~A} / \mathrm{W}$ & - & - & $1.7 \mathrm{~Hz}$ & - & PC & MHP & $\begin{array}{l}\text { Large area, } \\
\text { flexible }\end{array}$ \\
\hline 2014 & 67 & $\begin{array}{l}\text { UV- } \\
\text { VIS }\end{array}$ & $0.3 \mathrm{~A} / \mathrm{W}$ & - & $8 \cdot 10^{13}$ & $3 \mathrm{MHz}$ & $100 \mathrm{~dB}$ & PD & MHP & High sensitivity \\
\hline 2015 & 56 & $\begin{array}{l}\text { VIS- } \\
1.1 \mathrm{um}\end{array}$ & EQE $80 \%$ & $2 \cdot 10^{13}$ & & $1 \mathrm{MHz}$ & - & PD & $\begin{array}{l}\text { CQD } \\
(\mathrm{PbS})\end{array}$ & Quantum funnel \\
\hline 2015 & 88 & $\begin{array}{l}\text { VIS- } \\
\text { NIR }\end{array}$ & $G=10$ & - & & $35 \mathrm{kHz}$ & - & PT & $\begin{array}{l}\text { CQD } \\
(\mathrm{PbS})\end{array}$ & $\begin{array}{l}\text { Phototransistor, } \\
\text { break dark-photo } \\
\text { gain compromise }\end{array}$ \\
\hline 2015 & 5 & $\begin{array}{l}\text { Red } \\
\text { and } \\
\text { NIR }\end{array}$ & $0.1 \mathrm{~A} / \mathrm{W}$ & $5 \cdot 10^{12}$ & - & $90 \mathrm{kHz}$ & $160 \mathrm{~dB}$ & PD & Polymer & $\begin{array}{l}\text { Color selectivity, } \\
\text { charge collection } \\
\text { narrowing }(\mathrm{CCN})\end{array}$ \\
\hline 2015 & 32 & VIS & $0.3 \mathrm{~A} / \mathrm{W}$ & $2 \cdot 10^{13}$ & & $50 \mathrm{kHz}$ & $100 \mathrm{~dB}$ & PD & Polymer & Fully printed \\
\hline 2015 & 118 & NIR & $0.12 \mathrm{~A} / \mathrm{W}$ & - & $10^{12}$ & - & - & PD & $\begin{array}{l}\text { Organic } \\
\text { dye }\end{array}$ & $\begin{array}{l}\text { Transparent, NIR } \\
\text { sensitivity }\end{array}$ \\
\hline 2015 & 66 & $\begin{array}{l}\text { UV- } \\
\text { VIS }\end{array}$ & $0.25 \mathrm{~A} / \mathrm{W}$ & $3 \cdot 10^{12}$ & & $450 \mathrm{kHz}$ & $230 \mathrm{~dB}$ & PD & MHP & $\begin{array}{l}\text { Increased BW } \\
\text { using buffer layer }\end{array}$ \\
\hline 2015 & 119 & $\begin{array}{l}\text { UV- } \\
\text { VIS }\end{array}$ & $0.3 \mathrm{~A} / \mathrm{W}$ & $8 \cdot 10^{12}$ & & - & $190 \mathrm{~dB}$ & PD & MHP & Low noise \\
\hline
\end{tabular}




\begin{tabular}{|c|c|c|c|c|c|c|c|c|c|c|}
\hline 2015 & 68 & $\begin{array}{l}\text { UV- } \\
\text { VIS }\end{array}$ & $0.4 \mathrm{~A} / \mathrm{W}$ & $10^{12}$ & - & $290 \mathrm{kHz}$ & $80 \mathrm{~dB}$ & PD & MHP & $\begin{array}{l}\text { Low noise and } \\
\text { stability }\end{array}$ \\
\hline 2015 & 72 & $\begin{array}{l}\text { UV- } \\
\text { VIS }\end{array}$ & $G \sim 300$ & $\begin{array}{c}\text { NEP } \\
0.18 \mathrm{pW}\end{array}$ & - & $8 \mathrm{kHz}$ & $170 \mathrm{~dB}$ & PD & MHP & $\begin{array}{l}\text { High gain through } \\
\text { trap engineering }\end{array}$ \\
\hline 2015 & 74 & X-ray & $\begin{array}{l}2 \cdot 10^{4} \\
\text { carrier } \\
\text { /photon }\end{array}$ & $\begin{array}{c}25 \\
\mathrm{mGy} / \mathrm{cm}^{2}\end{array}$ & - & $3 \mathrm{GHz}$ & - & PD & MHP & X-Ray sensor \\
\hline 2015 & 112 & $\begin{array}{l}\text { UV- } \\
\text { VIS }\end{array}$ & $5 \mathrm{~mA} / \mathrm{W}$ & $2 \cdot 10^{10}$ & - & $600 \mathrm{~Hz}$ & - & PD & MHP & $\begin{array}{l}\text { Narrowband } \\
\text { response through } \\
\mathrm{CCN} \text { in single } \\
\text { crystals }\end{array}$ \\
\hline 2015 & 73 & $\begin{array}{l}\text { UV- } \\
\text { VIS }\end{array}$ & $0.1 \mathrm{~A} / \mathrm{W}$ & $2 \cdot 10^{11}$ & & $0.3 \mathrm{MHz}$ & $120 \mathrm{~dB}$ & PD & MHP & $\begin{array}{l}\text { Narrowband } \\
\text { response through } \\
\text { doping/CCN }\end{array}$ \\
\hline 2016 & 90 & $\begin{array}{l}\text { UV- } \\
\text { NIR }\end{array}$ & $3 \cdot 10^{5} \mathrm{~A} / \mathrm{W}$ & $2.5 \cdot 10^{12}$ & & $100 \mathrm{~Hz}$ & & $\mathrm{PT}$ & $\begin{array}{c}\mathrm{CQD}+ \\
\mathrm{MoS}_{2}\end{array}$ & $\begin{array}{l}\text { Interface } \\
\text { passivation in } \\
\text { hybrid } \\
\text { phototransistors }\end{array}$ \\
\hline 2016 & 91 & $\begin{array}{l}\text { UV- } \\
\text { SWIR }\end{array}$ & $\begin{array}{l}\text { EQE 70- } \\
80 \%\end{array}$ & $4 \cdot 10^{12}$ & & $1.5 \mathrm{kHz}$ & $110 \mathrm{~dB}$ & $\begin{array}{l}\mathrm{PT} / \mathrm{P} \\
\mathrm{D}\end{array}$ & CQD-g & $\begin{array}{l}\text { Hybrid } \\
\text { photodiode/phototr } \\
\text { ansistor }\end{array}$ \\
\hline
\end{tabular}

$\dagger$ It is important to note that in several published works, the specific detectivity is inferred from the shot-noise $\left(S_{\mathrm{N}}=\left(2 \mathrm{q} I_{\mathrm{d}}\right)^{1 / 2}\right)$, neglecting thermal noise which is not trivial. This can lead to a severe overestimation of $D^{*}$ especially in nanostructured photodetector systems that are dominated by flicker noise $(1 / f)$ at frequencies below $1-10 \mathrm{kHz}$. 

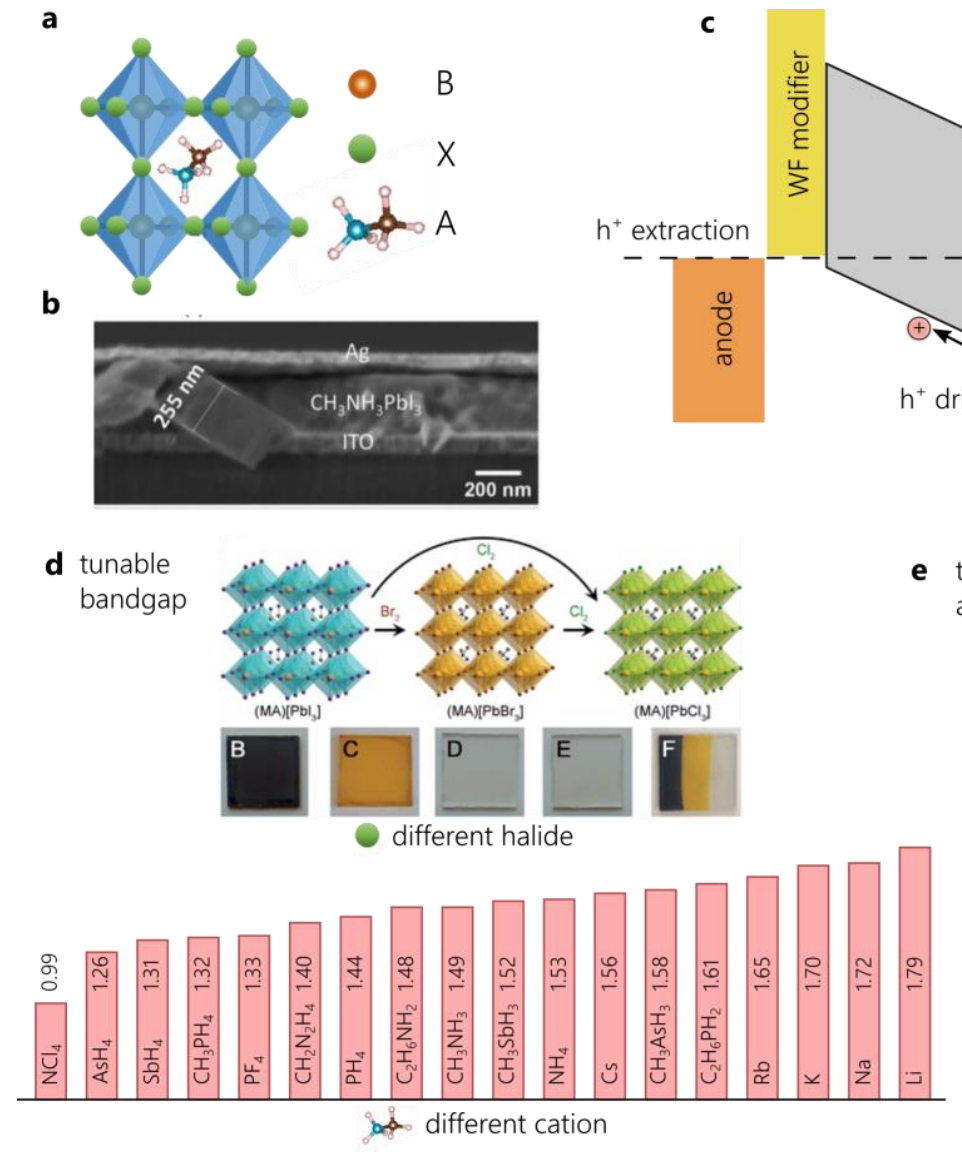

c

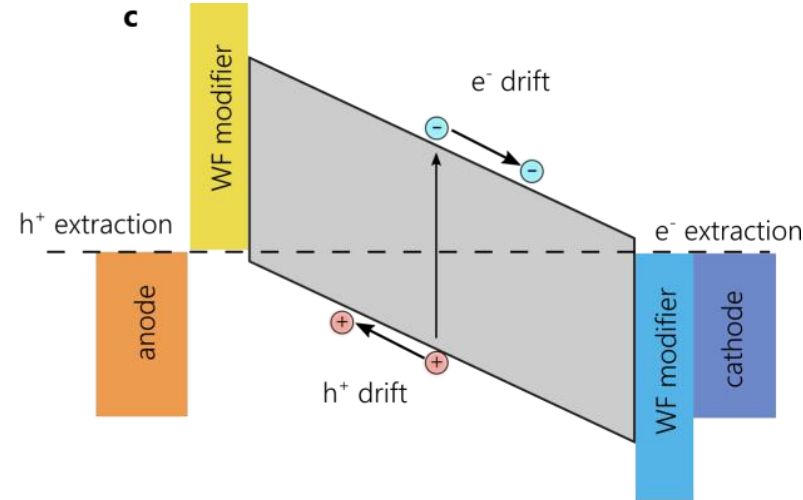

e trap engineering
and gain

$$
\text { and gain }
$$

Figure 2| Metal halide perovskite photodiodes. (a) Metal halide perovskites are class of hybrid organic-inorganic materials that possess a typical perovskite unit cell structure. They consists of two cations (A and B) and an $\mathrm{X}$ anion that bonds them together. (b) Cross-sectional scanning electron micrograph example of a methylammonium lead trihalide perovskite photodiode. (c) Principle of operation of organohalide perovskite photodiodes. The collection of photogenerated carriers is drift-assisted, towards selective anode and cathode electrodes. (d) Metal halide perovskite's bandgap can be widely tuned across the VIS-NIR by halide or cation modification. ${ }^{120,121}$ (e) Device and interface engineering can lead to functionalities typical of CQD photodetectors, such as internal gain. 
hybrid phototransistors

a

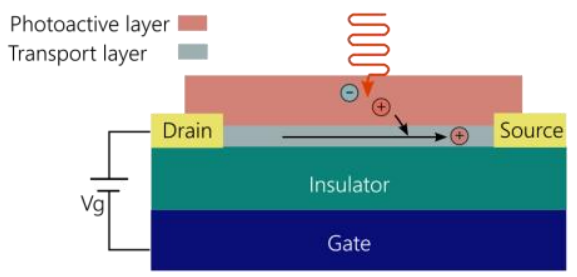

b

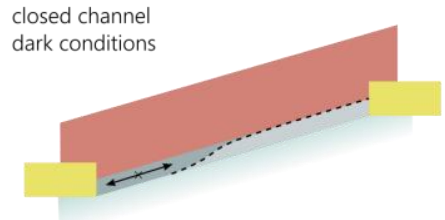

closed channel

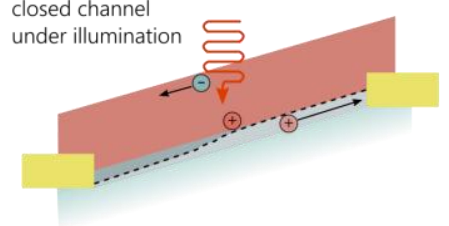

d

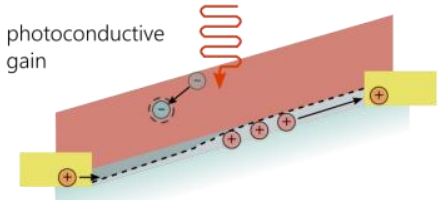

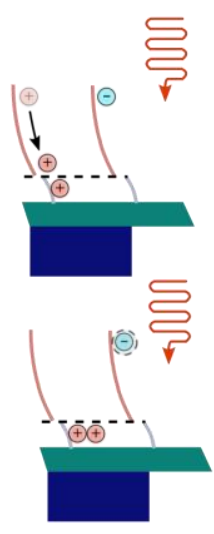

e

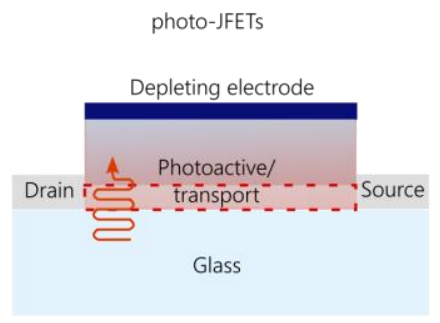

f dark conditions

fully depleted
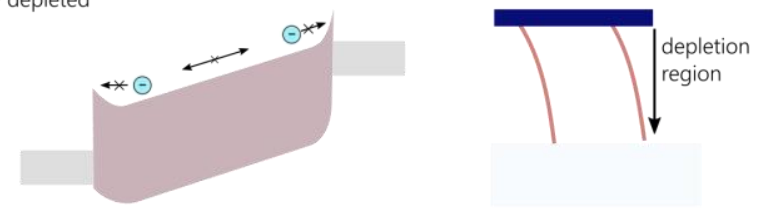

g under illumination open channel

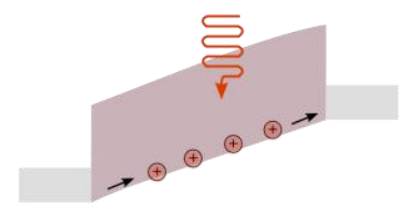

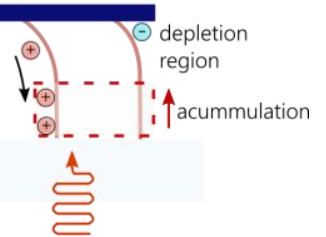

Figure 3 | Phototransistors, gain and transport. (a-d) Hybrid phototransistors separating electrical transport and optical sensitization. (a) Schematic of device operation. A transport channel is modulated by an external gate voltage $(\mathrm{Vg})$ and ideally closed under dark conditions (b). Under illumination (c), charges are generated in the photoactive material. (d) Depending on the gate voltage one type of carrier (holes in this case) is injected into the transporting medium, whereas the opposite one remains in the photoactive material. Given appropriate and favorable transport properties, injected charges can recirculate several times before recombination thus producing gain under illumination. (e-g) In photojunction Field-Effect Transistors (photoJFETs), a channel is formed under illumination, as the otherwise depleted material will not favor circulation between source and drain Schottky back-to-back electrodes. The use of this architecture provides gain while breaking prior photoconductors' response/speed/dark current trade-off. 

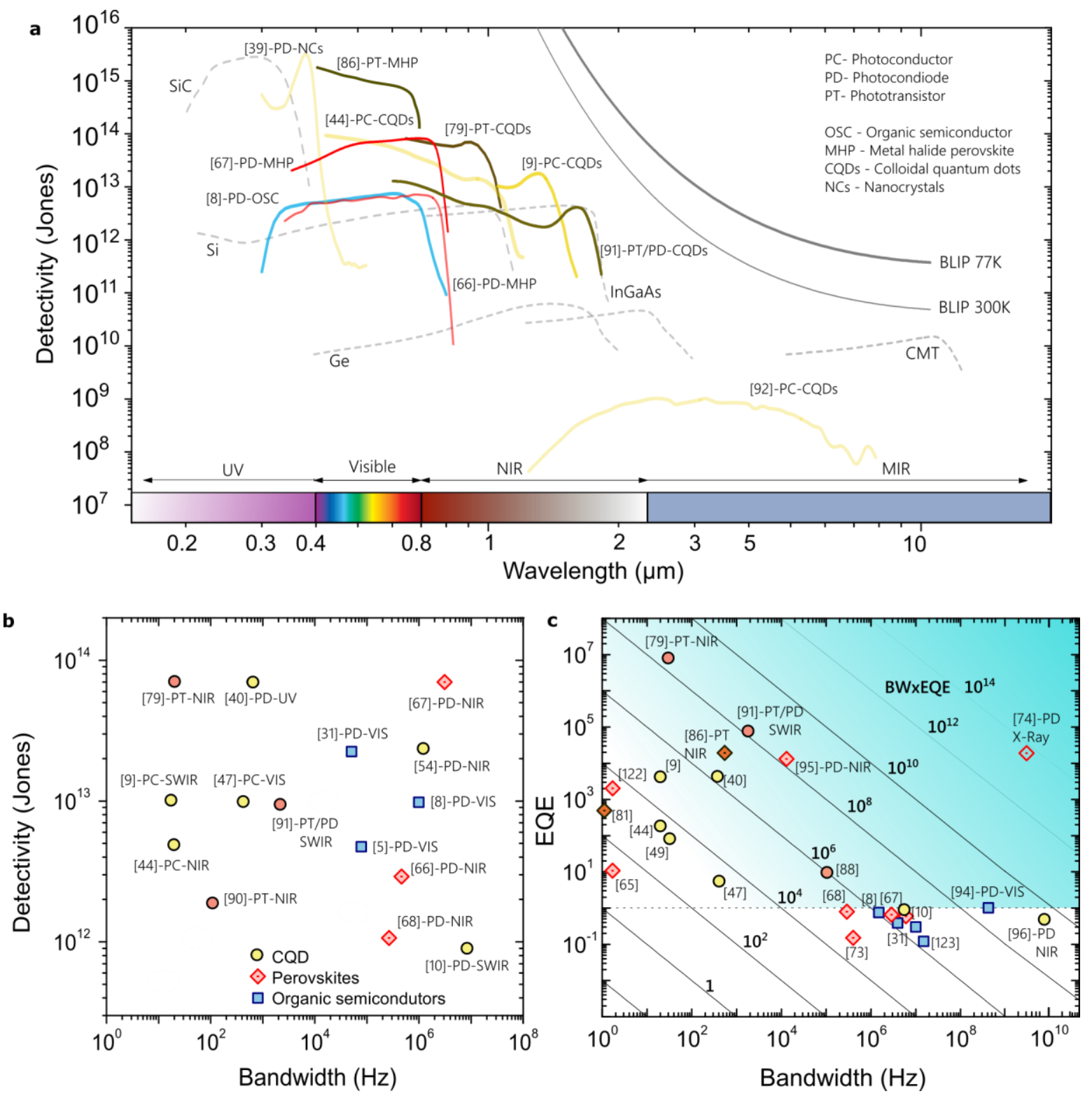

Figure 4 | Performance metrics of solution-processed photodetectors. (a) Specific detectivity of best solution processed photodetectors based on different materials and platforms compared to their best standard bulk reference counterparts (soft grey). Only measured $D^{*}$ are reported in this chart (b) Detectivity-bandwidth plot and (c) EQE-bandwidth plot of CQD, organic, and metal halide perovskite photodetectors illustrating the gain-noise-bandwidth compromise. The advent of metal halide perovskite photodetectors has resulted in significant improvements in visible sensitivities, as well as in $D^{*} \times B W$ and $\mathrm{EQE} \times B W$ products. Hybrid phototransistor and photoJFETs have also provided remarkable advances in that direction, surpassing the $10^{8} \mathrm{~Hz} \mathrm{EQE} \times B W$ limit. 


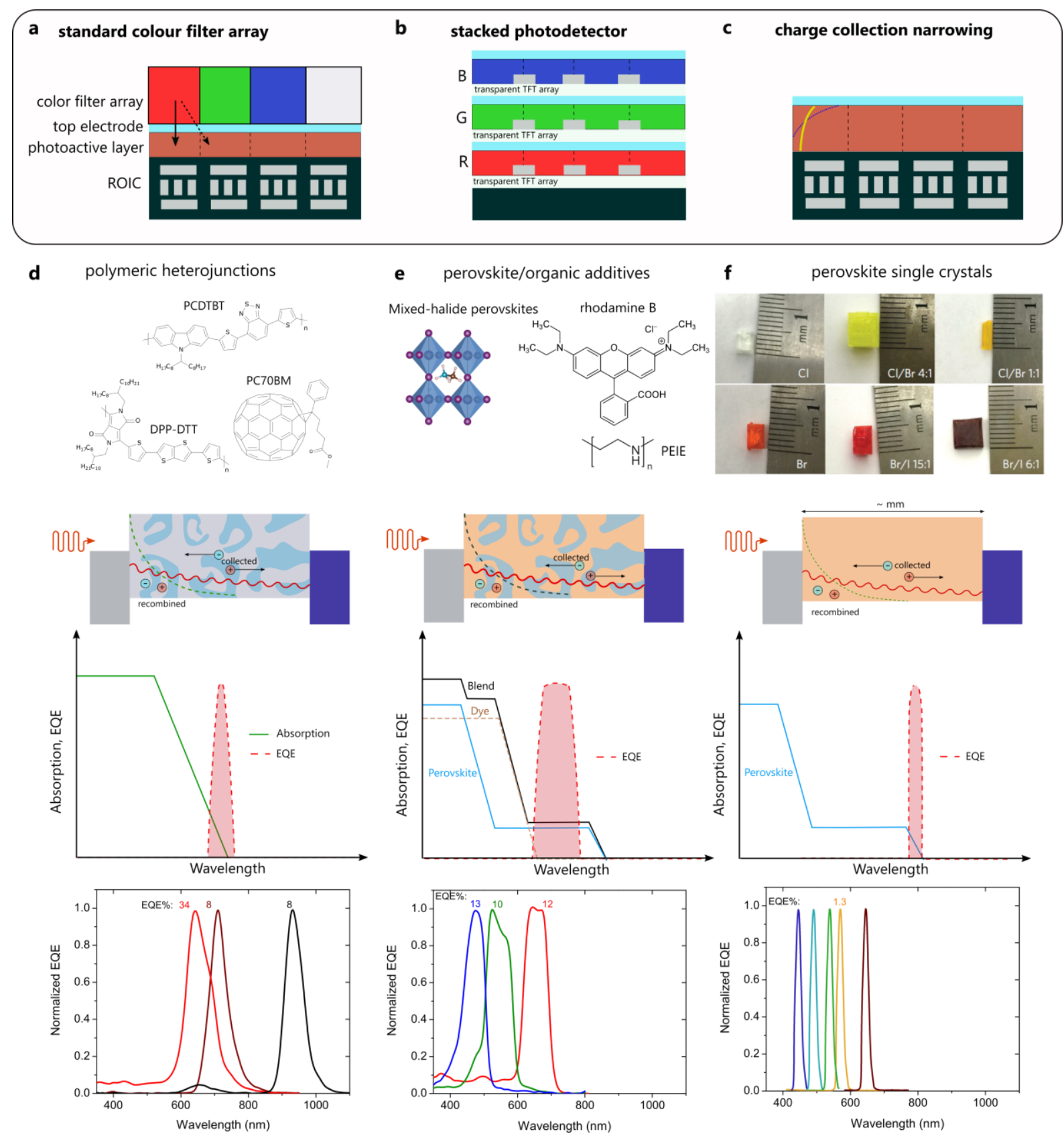

Figure 5 | Color selectivity and charge collection narrowing (CCN). Approaches for color selectivity: (a) standard colour filter array on top of a top-surface photodetector. The ultrathin nature of the photoactive film minimizes colour cross-over, but the presence of the colour-filter poses a limit in final sensor thickness. (b) Stacked photodetectors are attractive for they allow for a higher resolution. Their implementation is more complex since they would ultimately require the use of transparent thin film transistors; within this approach vertical cross-over needs to be carefully considered. (c) $\mathrm{CCN}$ can provide with ultra-narrowband photoresponse based on broadband absorbers. (d) Organic thick junction photodiodes. Within this approach, whereas surface generated electrons (corresponding to wavelengths of high absorption coefficient) must travel back to the cathode resulting in very slow extraction, photons 
corresponding to small absorption coefficients propagate through the material leading to interference and resulting in strong absorption and photogeneration in the volume. (e) The same concept can be applied for metal halide perovskite thick junction photodiodes, where the presence of ionic dyes reduces the crystallite size (in turn lowering the mobility and increasing the junction effective electrical thickness), and adjusting the absorption spectrum to the desired shape for CCN. (f) mm-thick single crystal organohalide perovskites can also be employed for ultranarrow $\mathrm{CCN}$ as a result of their low energetic disorder. 
a

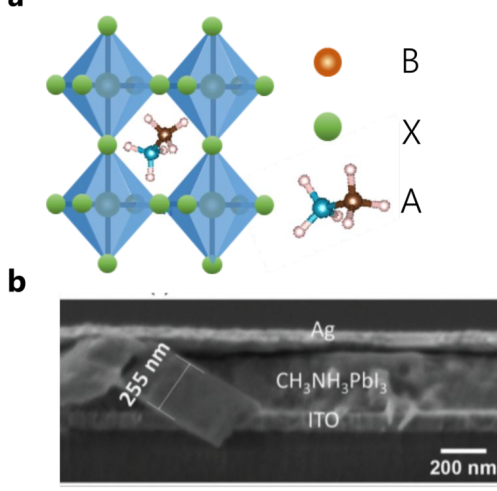

d tunable bandgap

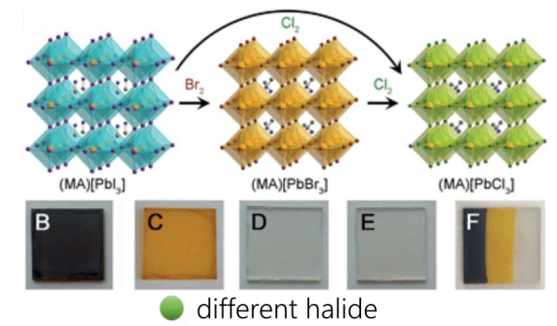

different halide$$
\text { 突 }
$$

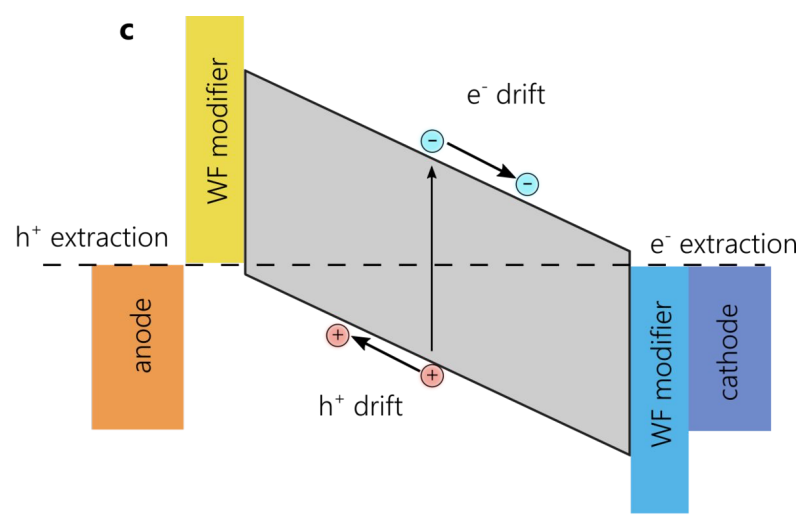

e trap engineering and gain

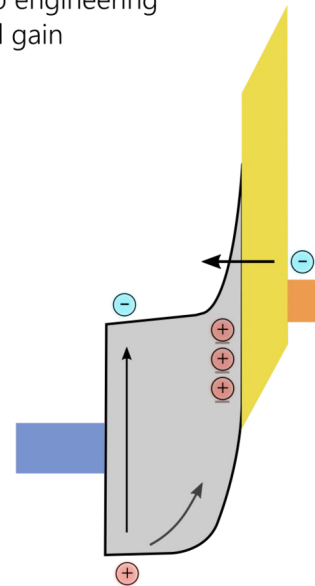




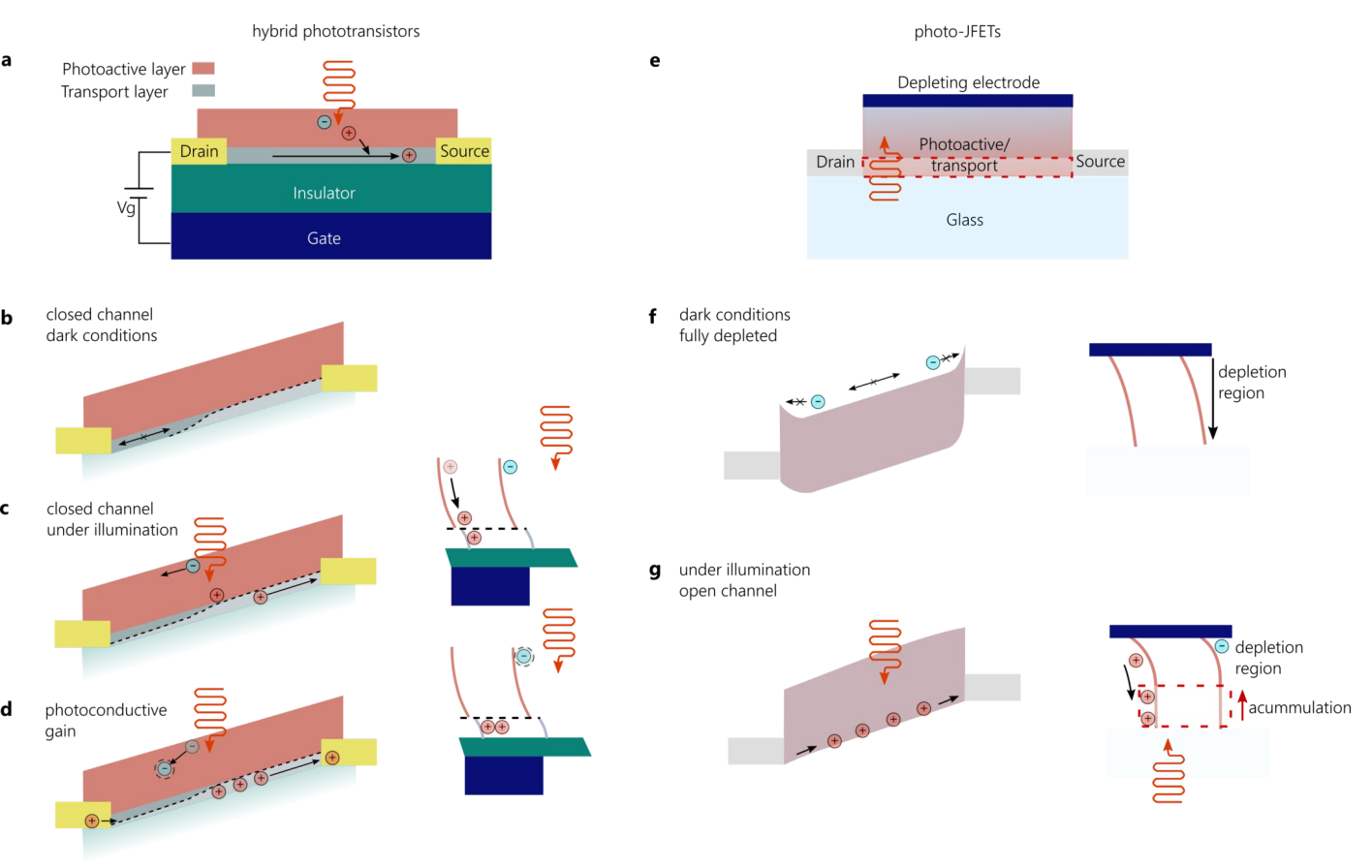



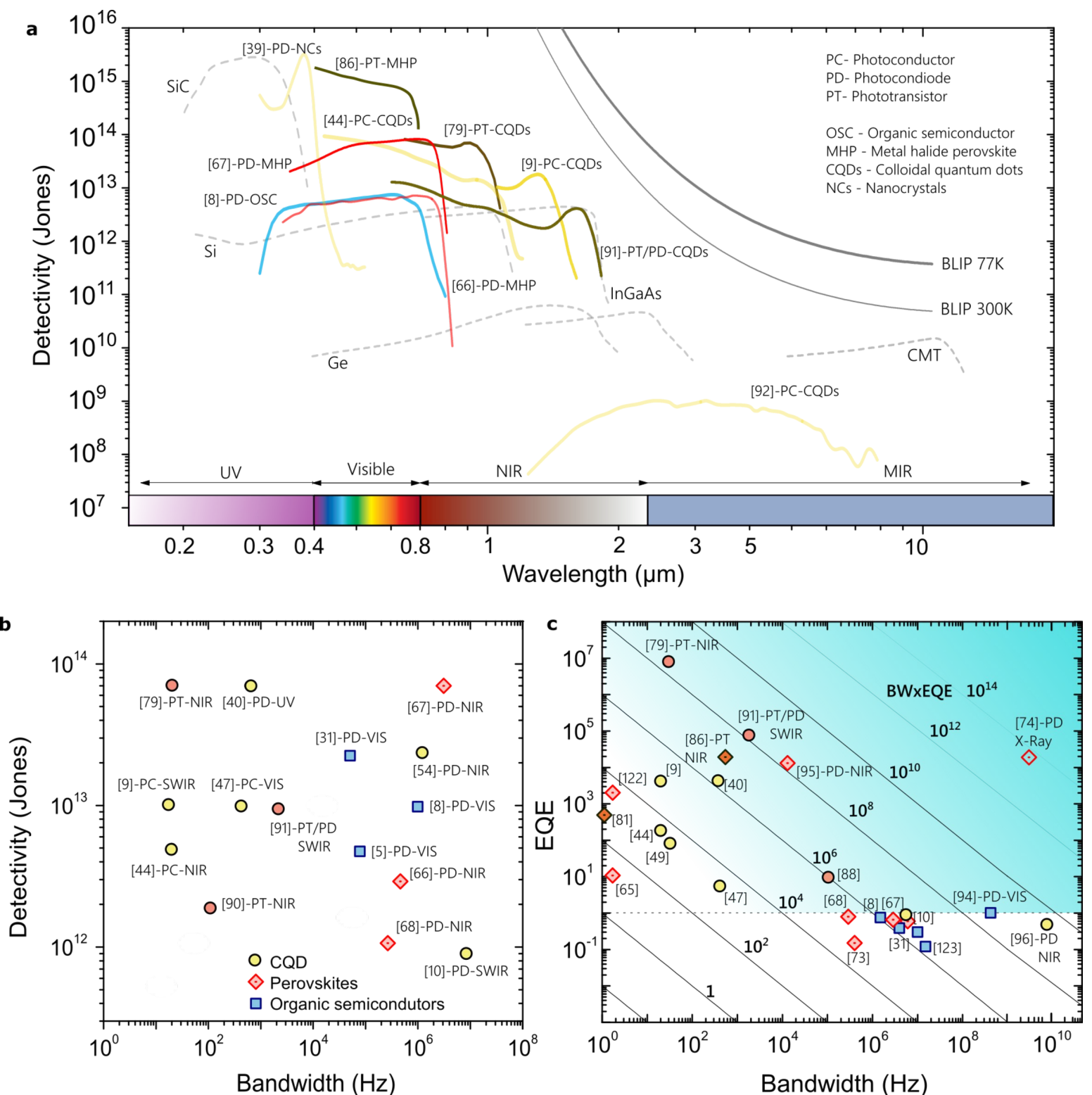

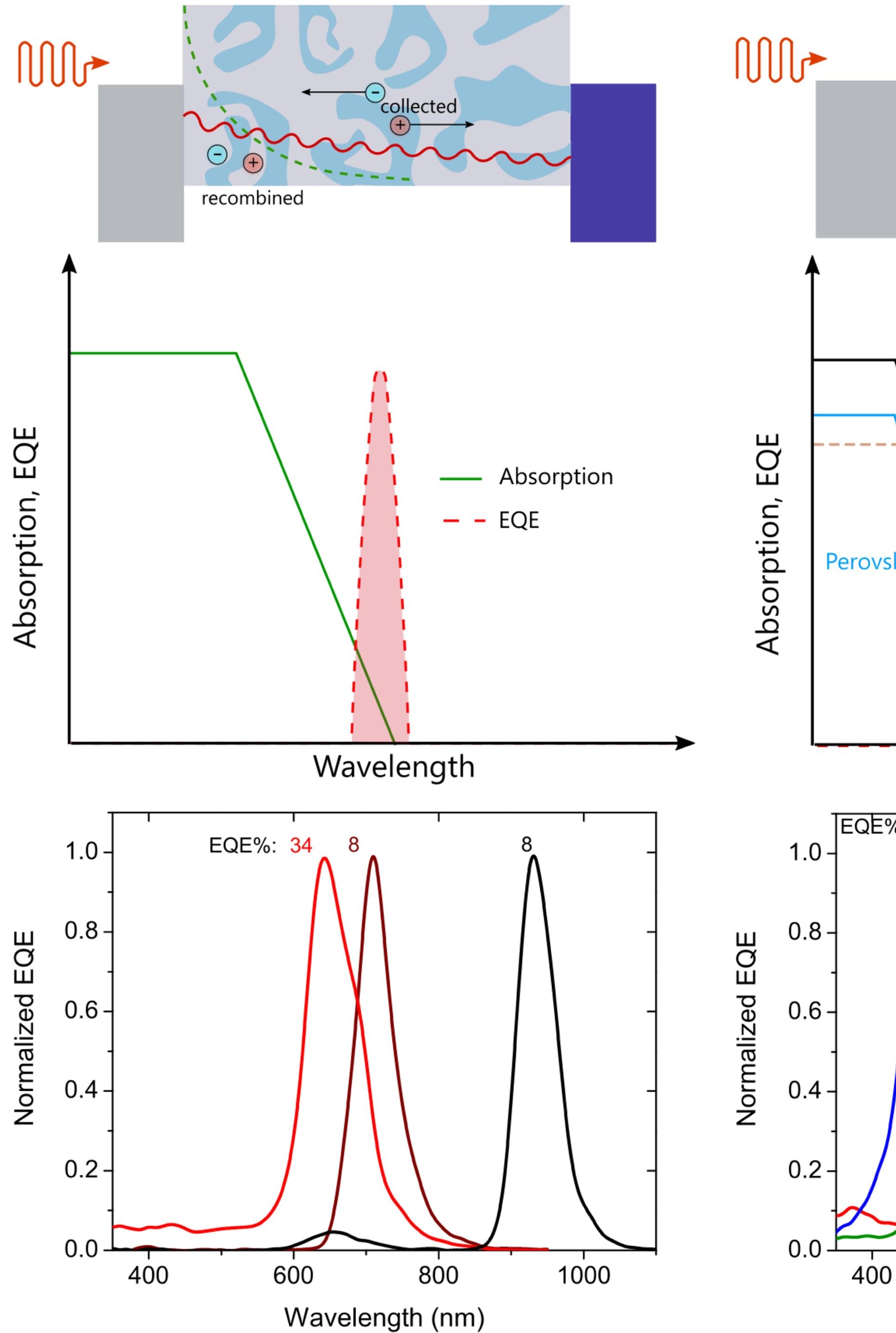